Electronic Supplementary Information for

\title{
Isolation and Characterization of a Homoleptic Tetramethylcobalt(III) Distorted Square- Planar Complex
}

\author{
Stephanie H. Carpenter, William W. Brennessel, and Michael L. Neidig* \\ Department of Chemistry, University of Rochester, Rochester, NY 14627 USA
}

Table of Contents

1. Experimental Procedures $\quad$ S2

$\begin{array}{ll}\text { 1.1. General considerations } & \text { S2 }\end{array}$

1.2. Preparation of $\left[\mathrm{Mg}(\mathrm{acac})(\mathrm{NMP})_{4}\right]\left[\mathrm{CoMe}_{4}\right](\mathbf{1}) \quad$ S2

1.3. Handling temperature sensitive crystals for single-crystal X-ray diffraction $\quad \mathrm{S} 2$

1.4. Collection of temperature sensitive crystalline material $\quad$ S3

1.5. Preparation of NMR samples $\quad$ S3

1.6. Preparation of low-temperature UV-Vis samples $\quad$ S3

1.7. Electron paramagnetic resonance (EPR) spectroscopy $\quad$ S3

1.8. Atomic absorption spectroscopy (AAS) S3

2. Supplementary Data $\quad$ S4

2.1. NMR spectra $\quad$ S4

2.2. Low-temperature UV-vis spectra $\quad$ S6

2.3. Comparisons to other homoleptic tetramethyl transition metal complexes $\quad \mathrm{S} 7$

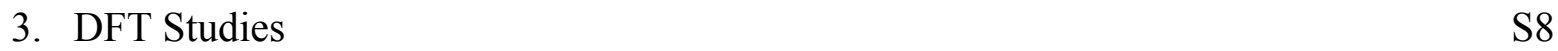

3.1. DFT experimental details $\quad$ S8

3.2. Spin state calculations $\quad$ S9

3.3. Charge decomposition analysis $\quad \mathrm{S} 10$

3.4. Selected orbital depictions of $\left[\mathrm{CoMe}_{4}\right]^{-} \quad \mathrm{S} 11$

4. References $\quad \mathrm{S} 12$

5. Single Crystal X-ray Diffraction Data $\quad$ S13

5.1. CCDC Deposition $\quad$ S13

5.2. $\left[\mathrm{Mg}(\mathrm{acac})(\mathrm{NMP})_{4}\right]\left[\mathrm{CoMe}_{4}\right](\mathbf{1}) \quad \mathrm{S} 14$

5.3. $\left[\mathrm{Mg}(\mathrm{acac})(\mathrm{NMP})_{4}\right]\left[\mathrm{CoMe}_{4}\right][\mathrm{Br}] \cdot 2 \mathrm{NMP} \quad \mathrm{S} 27$ 


\section{Experimental Procedures}

1.1 General considerations. All reagents were purchased from commercial sources. All air- and moisture-sensitive manipulations were carried out in an MBraun inert-atmosphere $\left(\mathrm{N}_{2}\right)$ glovebox equipped with a direct liquid nitrogen feed through inlet line. All anhydrous solvents were freshly dried using activated alumina/4 $\AA$ molecular sieves and stored under an inert atmosphere. ${ }^{1} \mathrm{H}$ NMR, ${ }^{13} \mathrm{C}$ NMR, and ${ }^{19} \mathrm{~F}$ NMR spectra were recorded on a Bruker $400 \mathrm{MHz}\left(400 \mathrm{MHz},{ }^{1} \mathrm{H} ; 100\right.$ $\mathrm{MHz},{ }^{13} \mathrm{C} ; 376 \mathrm{MHz},{ }^{19} \mathrm{~F}$ ) spectrometer. Low temperature UV-vis was collected at $193 \mathrm{~K}$ on an Agilent Cary 60 UV-Vis Spectrophotometer equipped with a CoolSpeK UV cryostat from Unisoku Scientific Instruments.

1.2 Preparation of $\left[\mathbf{M g}(\mathbf{a c a c})(\mathbf{N M P})_{4}\right]\left[\mathrm{CoMe}_{4}\right](\mathbf{1}) . \mathrm{Co}(\mathrm{acac})_{3}(25 \mathrm{mg}, 0.07 \mu \mathrm{mol})$ was dissolved in $2 \mathrm{~mL} \mathrm{Et}_{2} \mathrm{O}$ and NMP $(200 \mu \mathrm{L}, 30$ equiv) in a scintillation vial. The green solution was cooled to $-80{ }^{\circ} \mathrm{C}$, where $\mathrm{MeMgBr}(1.0 \mathrm{M}$ in THF, $350 \mu \mathrm{L}, 5$ equiv) was added dropwise over the course of $1 \mathrm{~min}$. The solution began to turn orange and was allowed to mix over the course of $20 \mathrm{~min}$ at $-80{ }^{\circ} \mathrm{C}$ at $500 \mathrm{rpm}$. The solution was then filtered through cold Celite to remove any unreacted $\mathrm{Co}(\mathrm{acac})_{3}$. The resulting orange solution was allowed to sit at $-80^{\circ} \mathrm{C}$ for 2 hours in effort to crash out any magnesium salts. The solution was then filtered through cold Celite, and $250 \mu \mathrm{L}$ cold pentane was added to the solution. The vial was sealed with Dow Corning ${ }^{\circledR}$ High Vacuum Grease and stored at $-80{ }^{\circ} \mathrm{C}$. Crystals suitable for single crystal X-ray diffraction were observed after several days, and analogous crystals with different unit cells were observed. Note that 1 is extremely air-and temperature- sensitive, precluding further analyses. Additionally, crystalline magnesium salts are observed in conjunction to crystalline material of 1 , hence, a reliable percent yield could not be determined.

1.3 Handling temperature sensitive crystals for single crystal $X$-ray diffraction. Temperature sensitive crystals were transferred to a nitrogen purged glovebag in an aluminum pie block containing dry ice. A customized aluminum block containing inlet and outlet nozzles allowed for the passage of liquid nitrogen. The cold aluminum block was placed on the base of the microscope and was used to cool microscope slides containing SilOil. Aliquots of the cold crystalline material were placed on the cold microscope slides using liquid nitrogen cooled pipettes. Single crystals were transferred from the microscope slide using a goniometer pin that was encased in liquid nitrogen cooled aluminum.

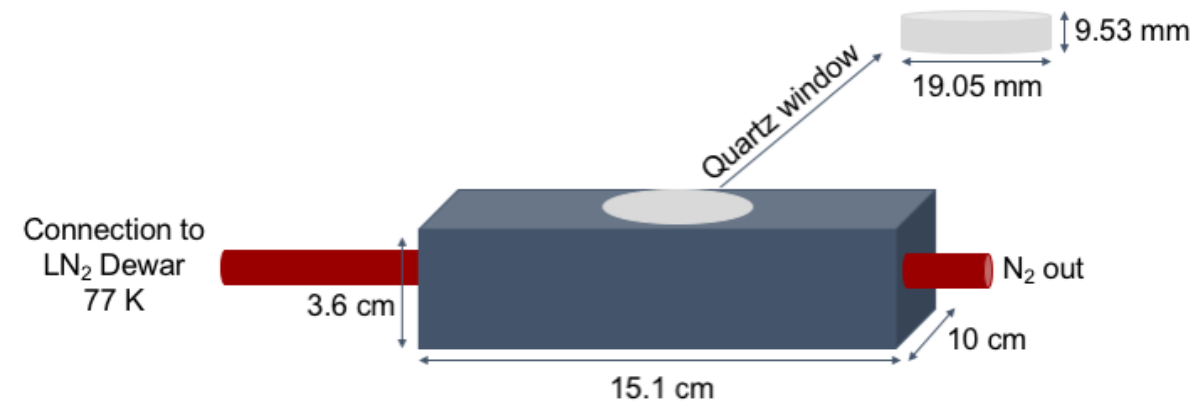

Figure S1. Depiction of aluminum cold block utilized for low temperature crystal work. 
1.4 Collection of temperature sensitive crystalline material. Crystalline material was grown in mother liquor as described above and brought into a glovebox via an aluminum pie block that had been cooled in liquid nitrogen. Crystalline material was immediately placed at $-80{ }^{\circ} \mathrm{C}$, and the mother liquor was removed using a cold (to liquid nitrogen temperatures) pipettes. The crystalline material was chilled in liquid nitrogen, and promptly collected using a cold spatula. The material was then dissolved in a known amount cold solvent (for solution samples).

1.5 Preparation of NMR samples. Crystalline material was collected as previously described. Crystalline material was dissolved in cold THF- $\mathrm{d}_{8}: \alpha, \alpha, \alpha$-trifluorotoluene $(1: 0.25)$. A cold pipette was used to transfer the dissolved material into a cold J-Young tube containing an internal standard of the same solvent mixture. The sample was then taped and placed on dry ice until placing in the instrument (which had been previously cooled to $-80^{\circ} \mathrm{C}$ ).

1.6 Preparation of low-temperature UV-vis samples. Crystalline material was collected as previously described. Crystalline material was dissolved in cold THF. A cold pipette (cooled to liquid nitrogen temperatures) was used to transfer the dissolved material into a chilled $10 \mathrm{~mm}$ OD Spectrosil far UV quartz cuvette from Starna Cells and sealed in the glovebox and immediately transferred to dry ice.

1.7 Electron paramagnetic resonance (EPR) spectroscopy. Crystalline material was collected as previously described and dissolved in a known amount of THF at $-80{ }^{\circ} \mathrm{C}$. A cold pipette was then used to transfer the dissolved crystalline material to a precooled (in liquid nitrogen) $4 \mathrm{~mm}$ OD suprasil quartz EPR tube from Wilmad Labglass. The solution in the EPR tube was immediately frozen in liquid nitrogen. The remaining solution not used for the EPR sample was saved for AAS, so that spin integration of any EPR signal could be completed. All X-band EPR spectra were collected on a Bruker EMXplus spectrometer containing a 4119HS cavity and an Oxford ESR-900 helium flow cryostat. All EPR spectra were collected at $10 \mathrm{~K}, 9.38 \mathrm{GHz}$.

1.8 Atomic absorption spectroscopy (AAS). Atomic absorption spectroscopy (AAS) analysis was performed using a Shimadzu AAS 7000. All samples for AAS were prepared from a known volume of 1 dissolved in solvent. The solvent was then removed in vacuo. The remaining solid was dissolved in a known volume of $1 \% \mathrm{HNO}_{3}$ in high purity DI $18 \mathrm{M} \Omega \mathrm{H}_{2} \mathrm{O}$. A solution of $\mathrm{CoBr}_{2}$ in $1 \% \mathrm{HNO}_{3}$ in high purity DI $18 \mathrm{M} \Omega \mathrm{H}_{2} \mathrm{O}$ was used to create a calibration curve based on parameters provided in the Shimadzu AAS Cookbook. Results from the AAS experiment were then used to back calculate the amount of $\mathbf{1}$ present in a given sample. 


\section{Supplementary Data}

\subsection{NMR Spectra}

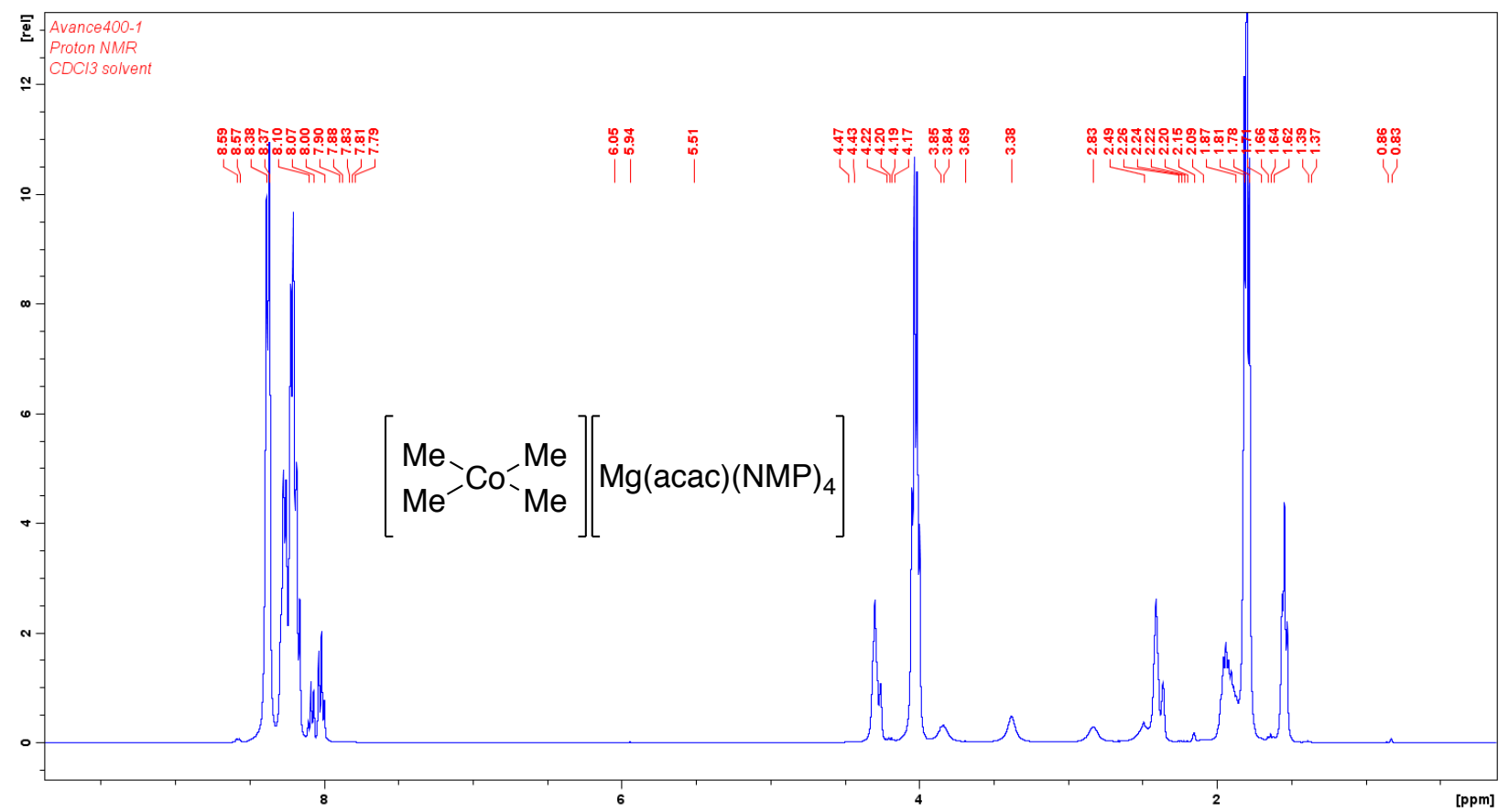

Figure S2. ${ }^{1} \mathrm{H}$ NMR of 1 in THF- $\mathrm{d}_{8}$ : trifluorotoluene at $193 \mathrm{~K}$.

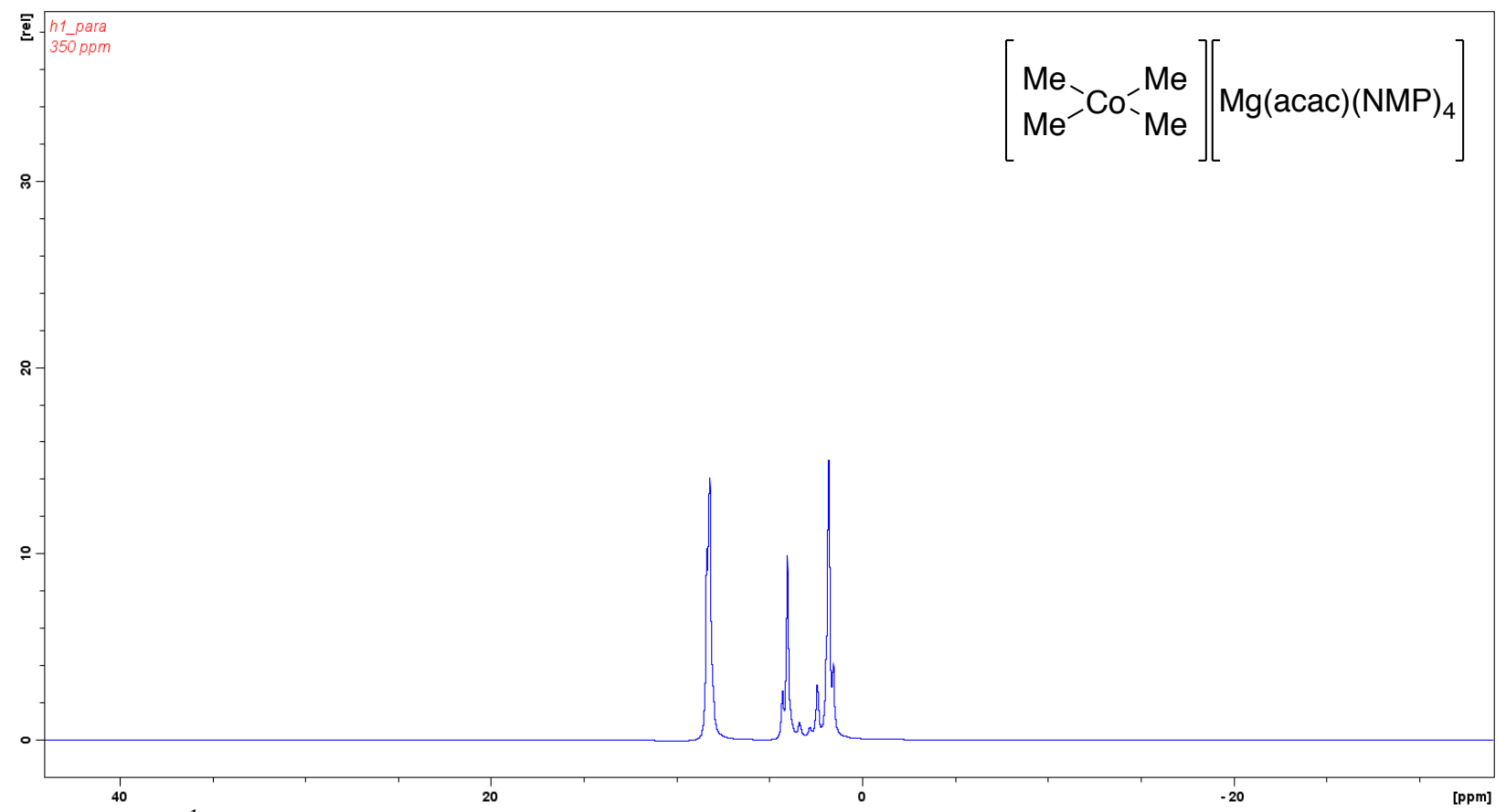

Figure S3. ${ }^{1} \mathrm{H}$ NMR of 1 in THF- $\mathrm{d}_{8}$ : trifluorotoluene at $193 \mathrm{~K}$ with a larger sweep width showing no peaks outside of the diamagnetic region. 


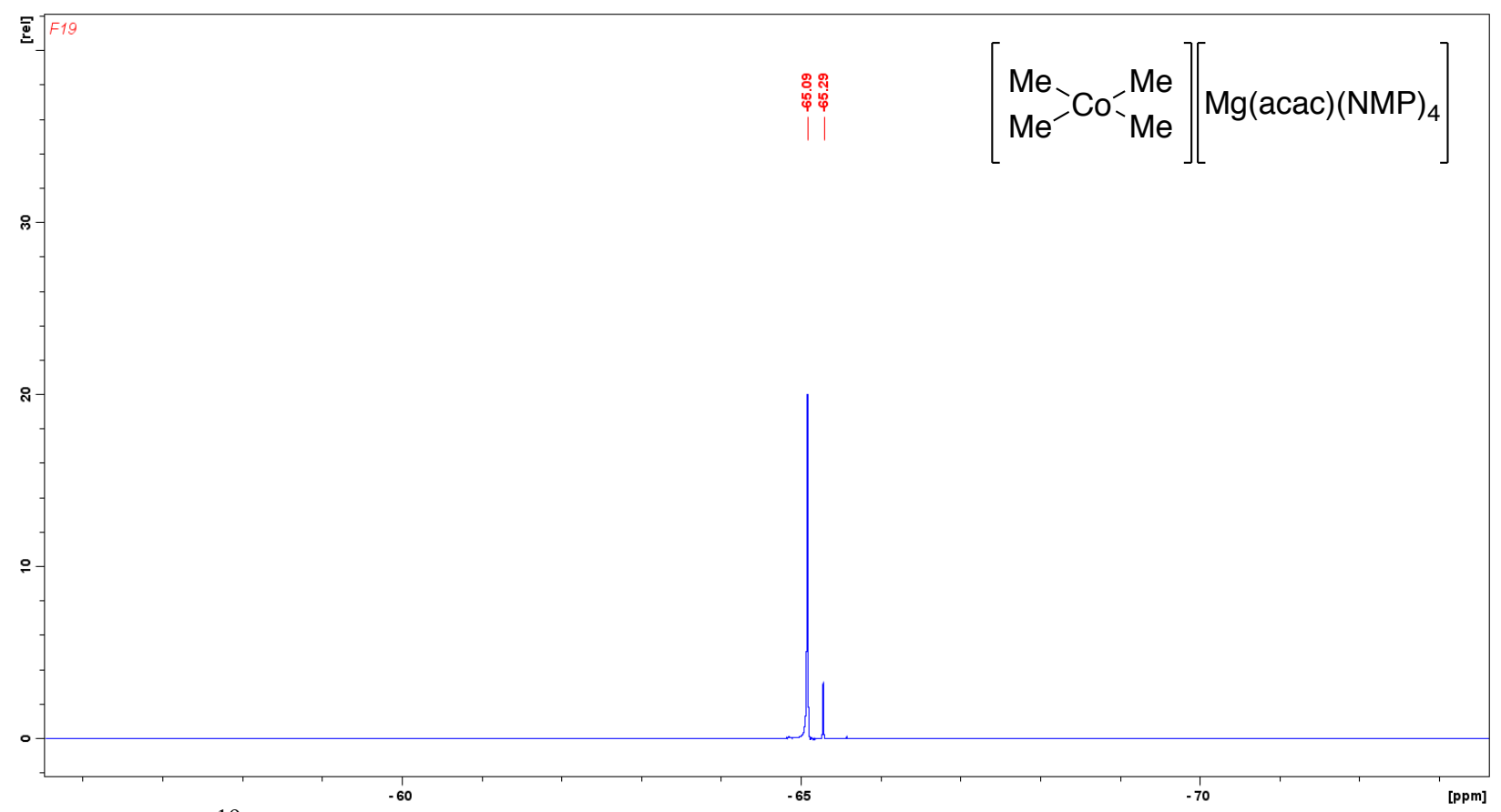

Figure S4. ${ }^{19} \mathrm{~F}$ Evan's Method NMR of $\mathbf{1}$ in THF- $\mathrm{d}_{8}$ : trifluorotoluene at $193 \mathrm{~K}$ with trifluorotoluene as the internal standard, where the difference between the internal standard and the external solvent is $\delta 0.2001 \mathrm{ppm}$. AAS showed the concentration of 1 to be $4.25 \mathrm{mM}$. 


\subsection{Low-temperature UV-Vis spectra}

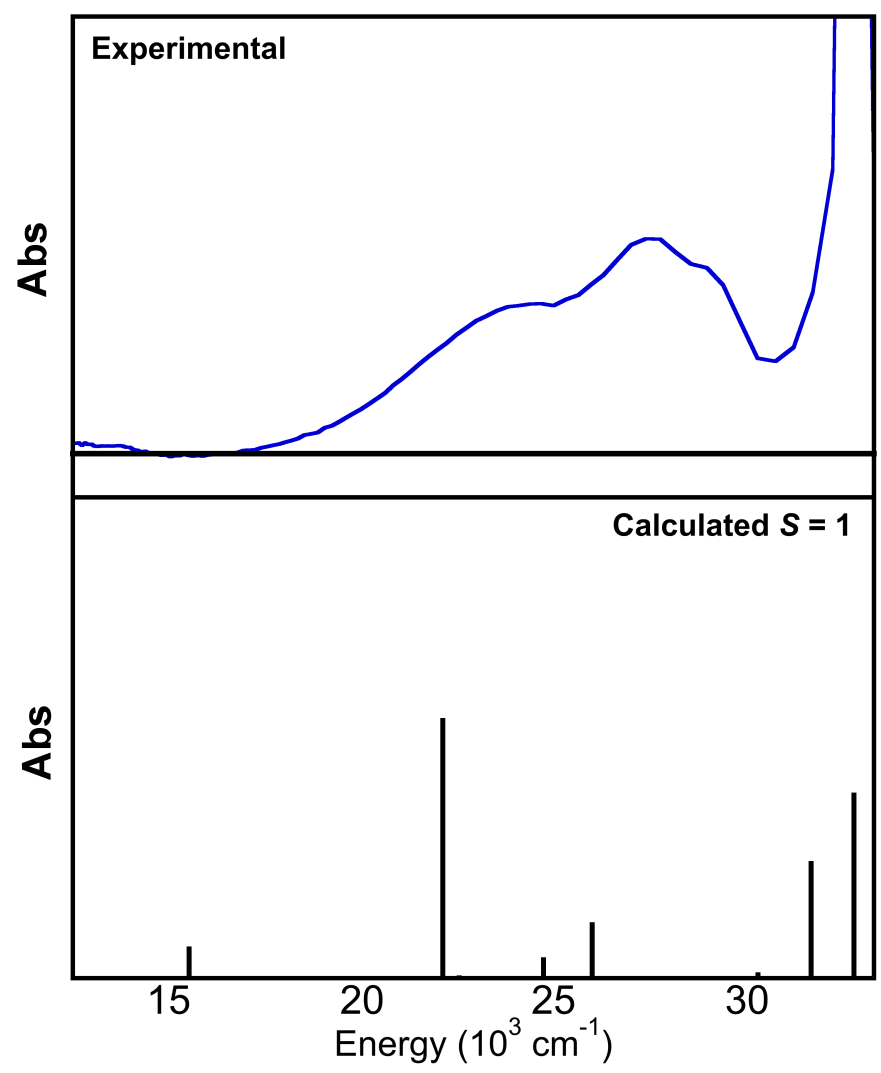

Figure S5. UV-Vis spectrum of 1 collected at $193 \mathrm{~K}$ (top, blue) and the simulated spectrum of $\mathbf{1}$ with $S=1$ calculated at $193 \mathrm{~K}$ (bottom). TD-DFT calculations were used to assign the observed transitions in the UV-vis electronic absorption spectrum, and the calculated TD-DFT transitions of $\mathrm{CoMe}_{4}{ }^{-}$correlate to the experimentally observed transitions. The transitions observed at $\sim 15500$ $\mathrm{cm}^{-1}$ and $\sim 22100 \mathrm{~cm}^{-1}$ have significant intraligand transitions with some mixed $\mathrm{d}$ character. The resulting transition around $\sim 24700 \mathrm{~cm}^{-1}$ is due to the mixing of d-d and MLCT character. The transitions at $\sim 26000 \mathrm{~cm}^{-1}$ and $\sim 30300 \mathrm{~cm}^{-1}$ exhibit significant mixing of MLCT, LMCT, and d-d character, as well. The transition observed $\sim 31700 \mathrm{~cm}^{-1}$ has significant $\mathrm{d}$-d character $\left(\beta 29, \mathrm{~d}_{\mathrm{xy}} \rightarrow\right.$ $\left.\beta 33, \mathrm{~d}_{\mathrm{xz}}\right)$. 


\subsection{Comparisons to other homoleptic tetramethyl transition metal complexes}

Table S1. Bond length $(\AA)$ and angle $\left(^{\circ}\right)$ comparison of 1 with other known tetramethyl transition metal complexes. Only the major position of $\mathbf{1}$ is used to compare bond lengths and angles in this table.

\begin{tabular}{|c|c|c|c|c|c|c|}
\hline & \multicolumn{7}{|c|}{ Transition Metal } \\
\hline & $\begin{array}{c}\text { Co } \\
\text { This work }\end{array}$ & $\begin{array}{c}\text { Fe } \\
\text { Ref: } 1\end{array}$ & $\begin{array}{c}\text { Au } \\
\text { Ref: } 2\end{array}$ & $\begin{array}{c}\text { Zn } \\
\text { Ref: } 3\end{array}$ & $\begin{array}{c}\text { Zn } \\
\text { Ref: } 3\end{array}$ & $\begin{array}{c}\text { Mn } \\
\text { Ref: } 4\end{array}$ \\
\hline Angles $\left(^{\circ}\right)$ & 93.09 & 89.71 & 90.83 & 114.62 & 111.67 & 90.89 \\
\hline & 93.52 & 91.12 & 89.17 & 114.62 & 111.67 & 91.75 \\
\hline & 94.11 & 91.35 & 90.83 & 114.62 & 111.67 & 92.41 \\
\hline & 92.13 & 94.75 & 89.17 & 114.62 & 111.67 & 92.13 \\
\hline & 156.46 & 158.68 & 180 & 99.6 & 105.15 & 161.77 \\
\hline & 147.85 & 161.00 & 180 & 99.6 & 105.15 & 160.82 \\
\hline Length $(\AA)$ & 1.959 & 2.042 & 2.103 & 2.07 & 2.16 & 2.084 \\
\hline & 2.001 & 2.051 & 2.096 & 2.07 & 2.16 & 2.085 \\
\hline & 1.952 & 2.009 & 2.103 & 2.07 & 2.16 & 2.067 \\
\hline & 2.010 & 2.042 & 2.096 & 2.07 & 2.16 & 2.075 \\
\hline
\end{tabular}




\section{DFT Studies}

3.1 DFT experimental details. Spin-unrestricted DFT calculations were performed using the Gaussian 09 package. ${ }^{5}$ All geometry optimizations were performed with either the B3LYP, PBEPBE, or PBE0 functional with the def2-TZVP basis set generated from basis set exchange on all atoms and inclusion of solvation effects using the polarized continuum model with THF as the solvent. ${ }^{6-12}$ The geometry of $\mathbf{1}$ was fully optimized starting from the X-ray crystal structures. All optimized geometries had positive frequencies. Molecular orbitals and TD-DFT calculations were performed using the B3LYP functional and def2-TZVP basis set on all atoms. The AOMix program was used to analyze MO compositions and Mayer bond orders. ${ }^{13,14}$ Orbitals from the Gaussian calculations were plotted with ChemCraft. TD-DFT was used to calculate the electronic transition energies for the 40 lowest energy states. Charge donation analysis (CDA) was performed using AOMix-FO. ${ }^{13}$ 


\subsection{Spin state calculations}

Table S2. Comparison of calculated structural parameters of $\mathbf{1}$ as a function of spin state and counterion with (A) B3LYP, (B) PBEPBE, and (C) PBE0.

\begin{tabular}{|c|c|c|c|c|c|c|c|}
\hline $\begin{array}{l}\text { Bond lengths } \\
(\AA) \text { and } \\
\text { angles }\left(^{\circ}\right)\end{array}$ & $\begin{array}{c}\text { Crystal } \\
\text { parameters } \\
\text { of } \mathbf{1} \\
\text { (major } \\
\text { position) } \\
\end{array}$ & \multicolumn{3}{|c|}{ B3LYP with counterion } & \multicolumn{3}{|c|}{ B3LYP without counterion } \\
\hline & & $S=0$ & $S=1$ & $S=2$ & $S=0$ & $S=1$ & $S=2$ \\
\hline $\mathrm{Co}-\mathrm{C} 1$ & $1.959(2)$ & 1.98991 & 2.01061 & 2.06331 & 2.02188 & 2.0245 & 2.08222 \\
\hline Co-C2 & $2.001(2)$ & 1.91466 & 2.02787 & 2.08247 & 1.91361 & 2.02381 & 2.08163 \\
\hline $\mathrm{Co}-\mathrm{C} 3$ & $1.952(2)$ & 2.04082 & 2.02898 & 2.09149 & 2.02248 & 2.0231 & 2.08215 \\
\hline Co-C4 & $2.010(3)$ & 1.91343 & 2.01601 & 2.08192 & 1.91434 & 2.02402 & 2.0825 \\
\hline $\mathrm{C} 1-\mathrm{Co}-\mathrm{C} 2$ & $93.09(12)$ & 93.693 & 93.146 & 112.179 & 95.284 & 93.107 & 112.051 \\
\hline $\mathrm{C} 2-\mathrm{Co}-\mathrm{C} 3$ & $93.52(12)$ & 95.683 & 92.146 & 113.115 & 95.26 & 93.338 & 112.076 \\
\hline $\mathrm{C} 3-\mathrm{Co}-\mathrm{C} 4$ & $94.21(13)$ & 95.42 & 93.077 & 111.184 & 95.323 & 93.209 & 111.876 \\
\hline $\mathrm{C} 4-\mathrm{Co}-\mathrm{C} 1$ & $92.13(13)$ & 93.872 & 92.81 & 112.682 & 95.251 & 93.181 & 111.88 \\
\hline $\mathrm{C} 1-\mathrm{Co}-\mathrm{C} 3$ & $156.46(13)$ & 164.508 & 154.45 & 103.663 & 162.905 & 152.688 & 104.531 \\
\hline C2-Co-C4 & $147.85(12)$ & 105.712 & 152.98 & 104.275 & 103.508 & 152.572 & 104.638 \\
\hline \multirow[t]{3}{*}{ Energy (A.U) } & & -3391.656 & -3391.679 & -3391.657 & -1542.389 & -1542.413 & -1542.391 \\
\hline & & \multicolumn{3}{|c|}{ PBEPBE with counterion } & \multicolumn{3}{|c|}{ PBEPBE without counterion } \\
\hline & & $S=0$ & $S=1$ & $S=2$ & $S=0$ & $S=1$ & $S=2$ \\
\hline Co-C1 & $1.959(2)$ & 1.97172 & 1.97921 & 2.05997 & 1.89218 & 1.99537 & 2.06617 \\
\hline $\mathrm{Co}-\mathrm{C} 2$ & $2.001(2)$ & 1.8898 & 2.00026 & 2.07039 & 1.99968 & 1.99435 & 2.06531 \\
\hline $\mathrm{Co}-\mathrm{C} 3$ & $1.952(2)$ & 2.01448 & 2.00182 & 2.05929 & 1.99909 & 1.99319 & 2.06588 \\
\hline Co-C4 & $2.010(3)$ & 1.88842 & 1.986 & 2.0653 & 1.89214 & 1.99438 & 2.06665 \\
\hline $\mathrm{C} 1-\mathrm{Co}-\mathrm{C} 2$ & $93.09(12)$ & 92.52 & 93.973 & 104.865 & 94.052 & 94.371 & 111.081 \\
\hline C2-Co-C3 & $93.52(12)$ & 97.688 & 94.535 & 114.632 & 166.439 & 94.688 & 106.678 \\
\hline C3-Co-C4 & $94.21(13)$ & 95.334 & 95.686 & 114.181 & 94.184 & 94.591 & 110.829 \\
\hline C4-Co-C1 & $92.13(13)$ & 93.713 & 93.621 & 103.145 & 105.897 & 94.359 & 106.266 \\
\hline C1-Co-C3 & $156.46(13)$ & 163.866 & 146.374 & 108.147 & 94.095 & 147.57 & 111.046 \\
\hline $\mathrm{C} 2-\mathrm{Co}-\mathrm{C} 4$ & $147.85(12)$ & 106.4 & 148.824 & 110.733 & 93.987 & 147.346 & 111.007 \\
\hline \multirow[t]{3}{*}{ Energy (A.U) } & & -3388.983 & -3388.996 & -3399.952 & -1541.896 & -1541.906 & -1541.874 \\
\hline & & \multicolumn{3}{|c|}{ PBE0 with counterion } & \multicolumn{3}{|c|}{ PBE0 without counterion } \\
\hline & & $S=0$ & $S=1$ & $S=2$ & $S=0$ & $S=1$ & $S=2$ \\
\hline $\mathrm{Co}-\mathrm{C} 1$ & $1.959(2)$ & 1.96377 & 1.9965 & 2.06171 & 1.99748 & 2.00775 & 2.05959 \\
\hline $\mathrm{Co}-\mathrm{C} 2$ & $2.001(2)$ & 1.89285 & 2.00865 & 2.0707 & 1.89221 & 2.00733 & 2.05895 \\
\hline Co-C3 & $1.952(2)$ & 2.02422 & 2.01368 & 2.05438 & 1.99797 & 2.00687 & 2.05953 \\
\hline Co-C4 & $2.010(3)$ & 1.89199 & 2.00331 & 2.04611 & 1.89284 & 2.00759 & 2.05977 \\
\hline $\mathrm{C} 1-\mathrm{Co}-\mathrm{C} 2$ & $93.09(12)$ & 93.049 & 91.699 & 111.482 & 94.912 & 91.948 & 112.127 \\
\hline C2-Co-C3 & $93.52(12)$ & 96.322 & 92.305 & 110.29 & 94.901 & 92.18 & 112.172 \\
\hline C3-Co-C4 & $94.21(13)$ & 95.645 & 92.439 & 111.1 & 94.927 & 92.042 & 111.89 \\
\hline C4-Co-C1 & $92.13(13)$ & 93.361 & 91.699 & 102.61 & 94.868 & 92.124 & 111.926 \\
\hline $\mathrm{C} 1-\mathrm{Co}-\mathrm{C} 3$ & $156.46(13)$ & 165.174 & 159.666 & 113.525 & 164.32 & 158.081 & 104.387 \\
\hline C2-Co-C4 & $147.85(12)$ & 103.193 & 156.773 & 107.45 & 102.425 & 158.06 & 104.551 \\
\hline Energy (A.U) & & -3389.096 & -3389.129 & -3389.104 & -1541.879 & -1541.909 & -1541.888 \\
\hline
\end{tabular}


Table S3. Comparison of calculated structural parameters of $\mathbf{1}$ as a function of temperature. Crystallographic data was collected at $100 \mathrm{~K}$.

\begin{tabular}{|c|c|c|c|c|c|}
\hline \multirow[t]{2}{*}{$\begin{array}{l}\text { Bond lengths } \\
(\AA) \text { and } \\
\text { angles }\left(^{\circ}\right)\end{array}$} & \multirow{2}{*}{$\begin{array}{c}\text { Crystal } \\
\text { parameters } \\
\text { of } \mathbf{1} \\
\text { (major } \\
\text { position) } \\
\end{array}$} & \multicolumn{2}{|c|}{ B3LYP with no counterion at $100 \mathrm{~K}$} & \multicolumn{2}{|c|}{ B3LYP without counterion at $0 \mathrm{~K}$} \\
\hline & & $S=0$ & $S=1$ & $S=0$ & $S=1$ \\
\hline $\mathrm{Co}-\mathrm{C} 1$ & $1.959(2)$ & 2.02189 & 2.02448 & 2.02188 & 2.0245 \\
\hline $\mathrm{Co}-\mathrm{C} 2$ & $2.001(2)$ & 1.91357 & 2.02377 & 1.91361 & 2.02381 \\
\hline $\mathrm{Co}-\mathrm{C} 3$ & $1.952(2)$ & 2.02265 & 2.02302 & 2.02248 & 2.0231 \\
\hline $\mathrm{Co}-\mathrm{C} 4$ & $2.010(3)$ & 1.91436 & 2.02398 & 1.91434 & 2.02402 \\
\hline $\mathrm{C} 1-\mathrm{Co}-\mathrm{C} 2$ & $93.09(12)$ & 95.279 & 93.112 & 95.284 & 93.107 \\
\hline $\mathrm{C} 2-\mathrm{Co}-\mathrm{C} 3$ & $93.52(12)$ & 95.278 & 93.347 & 95.26 & 93.338 \\
\hline $\mathrm{C} 3-\mathrm{Co}-\mathrm{C} 4$ & $94.21(13)$ & 95.301 & 93.230 & 95.323 & 93.209 \\
\hline $\mathrm{C} 4-\mathrm{Co}-\mathrm{C} 1$ & $92.13(13)$ & 95.254 & 93.200 & 95.251 & 93.181 \\
\hline $\mathrm{C} 1-\mathrm{Co}-\mathrm{C} 3$ & $156.46(13)$ & 162.908 & 152.621 & 162.905 & 152.688 \\
\hline $\mathrm{C} 2-\mathrm{Co}-\mathrm{C} 4$ & $147.85(12)$ & 105.512 & 152.522 & 103.508 & 152.572 \\
\hline Energy (A.U) & & -1542.363 & -1542.386 & -1542.389 & -1542.413 \\
\hline
\end{tabular}

Table S4. Comparison of calculated structural parameters of $\mathbf{1}$ in the gas phase and solvated.

\begin{tabular}{|c|c|c|c|c|c|}
\hline $\begin{array}{c}\text { Bond lengths } \\
(\AA) \text { and } \\
\text { angles }\left({ }^{\circ}\right)\end{array}$ & $\begin{array}{c}\text { Crystal } \\
\text { parameters } \\
\text { of 1 } \\
\text { major } \\
\text { position) }\end{array}$ & \multicolumn{2}{|c|}{$\begin{array}{c} \\
\text { B3LYP with no counterion in the gas } \\
\text { phase }\end{array}$} & \multicolumn{2}{c|}{ B3LYP without counterion in THF } \\
\hline & & $S=0$ & $S=1$ & $S=0$ & $S=1$ \\
\hline Co-C1 & $1.959(2)$ & 2.02188 & 2.0245 & 2.02257 & 2.02163 \\
\hline Co-C2 & $2.001(2)$ & 1.91361 & 2.02381 & 1.91563 & 2.02144 \\
\hline Co-C3 & $1.952(2)$ & 2.02248 & 2.0231 & 2.02280 & 2.02030 \\
\hline Co-C4 & $2.010(3)$ & 1.91434 & 2.02402 & 1.91658 & 2.02219 \\
\hline C1-Co-C2 & $93.09(12)$ & 95.284 & 93.107 & 95.110 & 93.340 \\
\hline C2-Co-C3 & $93.52(12)$ & 95.26 & 93.338 & 94.601 & 93.460 \\
\hline C3-Co-C4 & $94.21(13)$ & 95.323 & 93.209 & 95.501 & 93.308 \\
\hline C4-Co-C1 & $92.13(13)$ & 95.251 & 93.181 & 94.528 & 93.179 \\
\hline C1-Co-C3 & $156.46(13)$ & 162.905 & 152.688 & 164.059 & 151.963 \\
\hline C2-Co-C4 & $147.85(12)$ & 103.508 & 152.572 & 103.300 & 152.320 \\
\hline Energy (A.U) & & -1542.389 & -1542.413 & -1542.454 & -1542.476 \\
\hline
\end{tabular}

\subsection{Charge decomposition analysis}

Table S5. Mayer bond order and charge donation analysis for $\left[\mathrm{CoMe}_{4}\right]^{-}$.

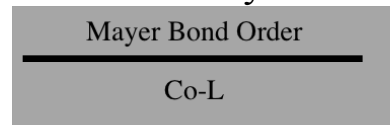

$0.578,0.578,0.578,0.578$

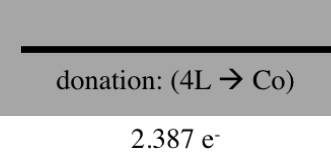

( $\left.\alpha: 1.184 \mathrm{e}^{-} ; \beta: 1.203 \mathrm{e}^{-}\right)$ charge donation analysis $(\alpha+\beta)$

back donation: $(\mathrm{Co} \rightarrow 4 \mathrm{~L})$

net charge donation to $\mathrm{Co}$

$0.132 \mathrm{e}^{-}$

$\left(\alpha: 0.044 \mathrm{e}^{-} ; \beta: 0.088 \mathrm{e}^{-}\right)$
$2.255 \mathrm{e}^{-}$ 


\subsection{Selected orbital depictions of $\left[\mathrm{CoMe}_{4}\right]^{-}$.}

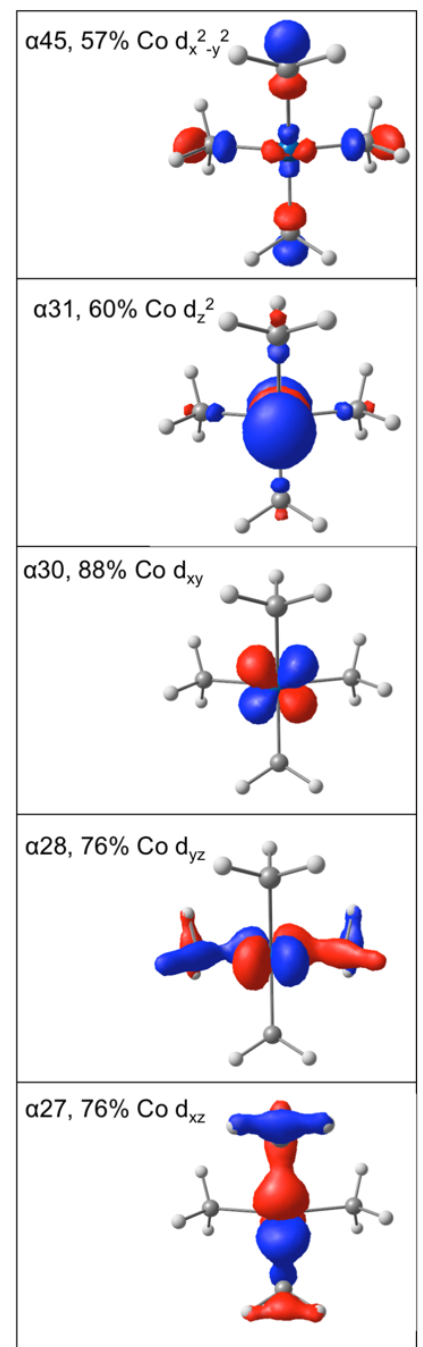

Figure S6. Selected $\alpha$-orbital depictions of 1 . 


\section{References}

1. Al-Afyouni, M. H.; Fillman, K. L.; Brennessel, W. W.; Neidig, M. L., Isolation and Characterization of a Tetramethyliron(III) Ferrate: An Intermediate in the Reduction Pathway of Ferric Salts with MeMgBr. J. Am. Chem. Soc. 2014, 136 (44), 15457-15460.

2. Zhu, D.; Lindeman, S. V.; Kochi, J. K., X-ray Crystal Structures and the Facile Oxidative (Au-C) Cleavage of the Dimethylaurate(I) and Tetramethylaurate(III) Homologs. Organometallics 1999, 18 (11), 2241-2248.

3. Weiss, E.; Wolfrum, R., Metal alkyl compounds. VI. Crystal structure of lithium tetramethylzincate. Chem. Ber. 1968, 101 (1), 35-40.

4. Morris, R. J.; Girolami, G. S., High-valent organomanganese chemistry. 1. Synthesis and characterization of manganese(III) and -(IV) alkyls. Organometallics 1991, 10 (3), 792-9.

5. Frisch, M. J. T., G. W.; Schlegel, H. B.; Scuseria, G. E.;Robb, M. A.; Cheeseman, J. R.; Scalmani, G.; Barone, V.; Mennucci,B.; Petersson, G. A.; Nakatsuji, H.; Caricato, M.; Li, X.; Hratchian, H.P.; Izmaylov, A. F.; Bloino, J.; Zheng, G.; Sonnenberg, J. L.; Hada, M.;Ehara, M.; Toyota, K.; Fukuda, R.; Hasegawa, J.; Ishida, M.; Nakajima,T.; Honda, Y.; Kitao, O.; Nakai, H.; Vreven, T.; Montgomery, J. A., Jr.;Peralta, J. E.; Ogliaro, F.; Bearpark, M.; Heyd, J. J.; Brothers, E.; Kudin,K. N.; Staroverov, V. N.; Kobayashi, R.; Normand, J.; Raghavachari, K.;Rendell, A.; Burant, J. C.; Iyengar, S. S.; Tomasi, J.; Cossi, M.; Rega,N.; Millam, M. J.; Klene, M.; Knox, J. E.; Cross, J. B.; Bakken, V.;Adamo, C.; Jaramillo, J.; Gomperts, R.; Stratmann, R. E.; Yazyev, O.;Austin, A. J.; Cammi, R.; Pomelli, C.; Ochterski, J. W.; Martin, R. L.;Morokuma, K.; Zakrzewski, $\quad$ V. $\quad$ G.; $\quad$ Voth, $\quad$ G. $\quad$ A.; $\quad$ Salvador, P.;Dannenberg,J.J.;Dapprich,S.;Daniels,A.D.;Farkas,Ö.;Foresman, J. B.; Ortiz, J. V.; Cioslowski, J.; Fox, D. J.Gaussian 09, Revision D.01; Gaussian, Inc.: Wallingford, CT, 2013.

6. Lee, C.; Yang, W.; Parr, R. G., Development of the Colle-Salvetti correlation-energy formula into a functional of the electron density. Phys. Rev. B: Condens. Matter Mater. Phys. 1988, 37 (2), 785-789.

7. Becke, A. D. J., A new mixing of Hartree-Fock and local-density-functional theories. J. Phys. Chem. 1993, 98 (2), 1372-1377.

8. Weigend, F.; Ahlrichs, R., Balanced basis sets of split valence, triple zeta valence and quadrupole zeta valence quality for H to Rn: Design and assessment of accuracy. Phys. Chem. Chem. Phys. 2005, 7 (18), 3297-3305.

9. Schaefer, A.; Huber, C., Ahlrichs, R., Fully optimized contracted Gaussian basis sets of triple zeta valence quality for atoms Li to Kr. J. Chem. Phys. 1994, 100 (8), 5829-5835.

10. Feller, D., The role of databases in support of computational chemistry calculations. J. Comput. Chem. 1996, 17 (13). 1571-1586.

11. Schuchardt, K. L.; Didier, B. T.; Elsethagen, T.; Sun, L.; Gurumoorthi, V.; Chase, J.; Li, J.; Windus, T. L., Basis Set Exchange: A Community Database for Computational Sciences. J. Chem. Inf. Model. 2007, 47 (3), 1045-1052.

12. Tomasi, J.;. Mennucci, B.; Cammi, R., Quantum Mechanical Continuum Solvation Models. Chem. Rev. 2005, 105 (8), 2999-3093.

13. Gorelsky, S. I. AO Mix: Program for Molecular Orbital Analysis, version 6.8.5; 2014; http://www.sgchem.net/.

14. Gorelsky, S. I.; Lever, A. B. P., Electronic structure and spectra of ruthenium diamine complexes by density functional theory and INDO/S. Comparison of the two methods. J. Organomet. Chem. 2001, 635 (1-2), 187196. 


\section{Single Crystal X-ray Diffraction Data}

5.1 CCDC Deposition. All crystal structures have been deposited with the Cambridge Crystallographic Data Center (CCDC). The crystal structures have been assigned the following CCDC deposition numbers:

$\left[\mathrm{Mg}(\mathrm{acac})(\mathrm{NMP})_{4}\right]\left[\mathrm{CoMe}_{4}\right](\mathbf{1}): 1921622$

$\left[\mathrm{Mg}(\mathrm{acac})(\mathrm{NMP})_{4}\right]\left[\mathrm{CoMe}_{4}\right][\mathrm{Br}] \cdot 2 \mathrm{NMP}: 1921623$ 


\section{$5.2\left[\mathrm{Mg}(\operatorname{acac})(\mathrm{NMP})_{2}\right]\left[\mathrm{CoMe}_{4}\right](1)$}

REFERENCE NUMBER: neisc68

CRYSTAL STRUCTURE REPORT

$\mathrm{C}_{29} \mathrm{H}_{55} \mathrm{Co} \mathrm{Mg} \mathrm{N}_{4} \mathrm{O}_{6}$

or

$\left[\mathrm{Mg}(\mathrm{acac})(\mathrm{NMP})_{4}\right]\left[\mathrm{CoMe}_{4}\right]$

Report prepared for:

S. Carpenter, Prof. M. Neidig

July 18, 2018

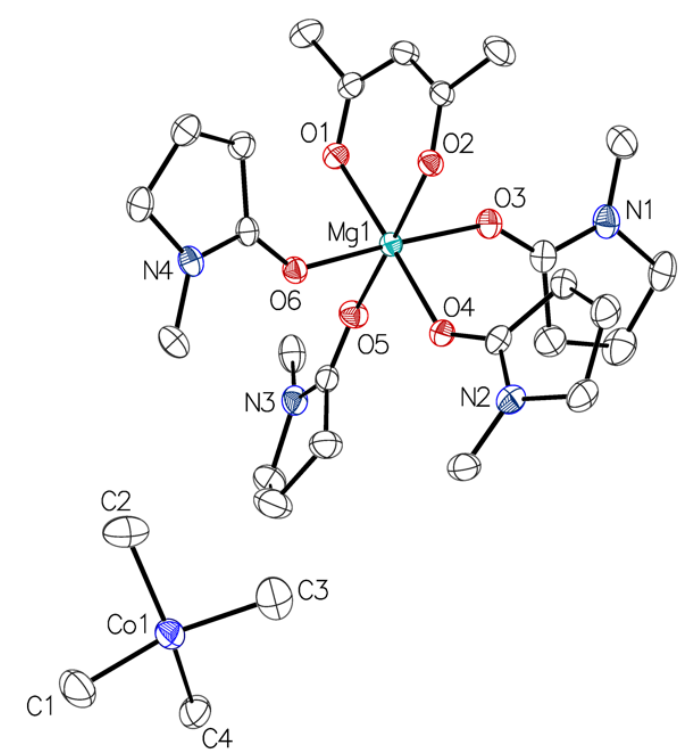

\footnotetext{
William W. Brennessel

X-ray Crystallographic Facility

Department of Chemistry, University of Rochester

120 Trustee Road

Rochester, NY 14627
} 


\section{Data collection}

A crystal $\left(0.276 \times 0.189 \times 0.119 \mathrm{~mm}^{3}\right)$ was placed onto a thin glass optical fiber or a nylon loop and mounted on a XtaLab Synergy-S Dualflex diffractometer equipped with a HyPix-6000HE HPC area detector for data collection at 100.01(10) K. A preliminary set of cell constants and an orientation matrix were calculated from a small sampling of reflections. ${ }^{1}$ A short pre-experiment was run, from which an optimal data collection strategy was determined. The full data collection was carried out using a PhotonJet $(\mathrm{Cu})$ X-ray Source with frame times of 0.36 and 1.46 seconds and a detector distance of $31.2 \mathrm{~mm}$. Series of frames were collected in $0.50^{\circ}$ steps in $\omega$ at different $2 \theta$, $\kappa$, and $\phi$ settings. After the intensity data were corrected for absorption, the final cell constants were calculated from the xyz centroids of 18036 strong reflections from the actual data collection after integration. ${ }^{1}$ See Table S6 for additional crystal and refinement information.

\section{Structure solution and refinement}

The structure was solved using ShelXT ${ }^{2}$ and refined using ShelXL. ${ }^{3}$ The space group $P-1$ was determined based on intensity statistics. Most or all non-hydrogen atoms were assigned from the solution. Full-matrix least squares / difference Fourier cycles were performed which located any remaining non-hydrogen atoms. All non-hydrogen atoms were refined with anisotropic displacement parameters. All hydrogen atoms were placed in ideal positions and refined as riding atoms with relative isotropic displacement parameters. The final full matrix least squares refinement converged to $R 1=0.0437\left(F^{2}, I>2 \sigma(I)\right)$ and $w R 2=0.1272\left(F^{2}\right.$, all data).

\section{Structure description}

The structure is the one suggested. The asymmetric unit contains one monocationic magnesium complex and one monoanionic cobalt complex in general positions. Methyl ligand C4 is modeled as disordered over two positions (0.73:0.27). The angle between the C1-Co1-C2 and C3-Co1-C4 planes is 39.0(1) degrees (that between planes C1Co1-C2 and C3-Co1-C4' is 43.8(4) degrees).

Structure manipulation and figure generation were performed using Olex $2 .{ }^{4}$ Unless noted otherwise all structural diagrams containing thermal displacement ellipsoids are drawn at the $50 \%$ probability level.

Data collection, structure solution, and structure refinement were conducted at the X-ray Crystallographic Facility, B04 Hutchison Hall, Department of Chemistry, University of Rochester. The instrument was purchased with funding from NSF MRI program grant CHE-1725028. All publications arising from this report MUST either 1) include William W. Brennessel as a coauthor or 2) acknowledge William W. Brennessel and the X-ray Crystallographic Facility of the Department of Chemistry at the University of Rochester. 
1 CrysAlisPro, version 171.39.46; Rigaku Corporation: Oxford, UK, 2018.

2 Sheldrick, G. M. SHELXT, version 2018/2; Acta. Crystallogr. 2015, A71, 3-8.

3 Sheldrick, G. M. SHELXL, version 2018/3; Acta. Crystallogr. 2015, C71, 3-8.

4 Dolomanov, O. V.; Bourhis, L. J.; Gildea, R. J.; Howard, J. A. K.; Puschmann, H. Olex2, version 1.2-ac3; J. Appl. Cryst. 2009, 42, 339-341.

Some equations of interest:

$$
\begin{gathered}
R_{\mathrm{int}}=\Sigma\left|F_{\mathrm{o}}^{2}-<F_{\mathrm{o}}^{2}>\right| / \Sigma\left|F_{\mathrm{o}}{ }^{2}\right| \\
R 1=\Sigma|| F_{\mathrm{o}}|-| F_{\mathrm{c}} \| / \Sigma\left|F_{\mathrm{o}}\right| \\
w R 2=\left[\Sigma\left[w\left(F_{\mathrm{o}}{ }^{2} F_{\mathrm{c}}{ }^{2}\right)^{2}\right] / \Sigma\left[w\left(F_{\mathrm{o}}{ }^{2}\right)^{2}\right]\right]^{1 / 2} \\
\text { where } w=1 /\left[\sigma^{2}\left(F_{\mathrm{o}}{ }^{2}\right)+(a P)^{2}+b P\right] \text { and } \\
P=1 / 3 \max \left(0, F_{\mathrm{o}}{ }^{2}\right)+2 / 3 F_{\mathrm{c}}{ }^{2} \\
\mathrm{GOF}=S=\left[\Sigma\left[w\left(F_{\mathrm{o}}{ }^{2}-F_{\mathrm{c}}{ }^{2}\right)^{2}\right] /(m-n)\right]^{1 / 2}
\end{gathered}
$$

where $m=$ number of reflections and $n=$ number of parameters
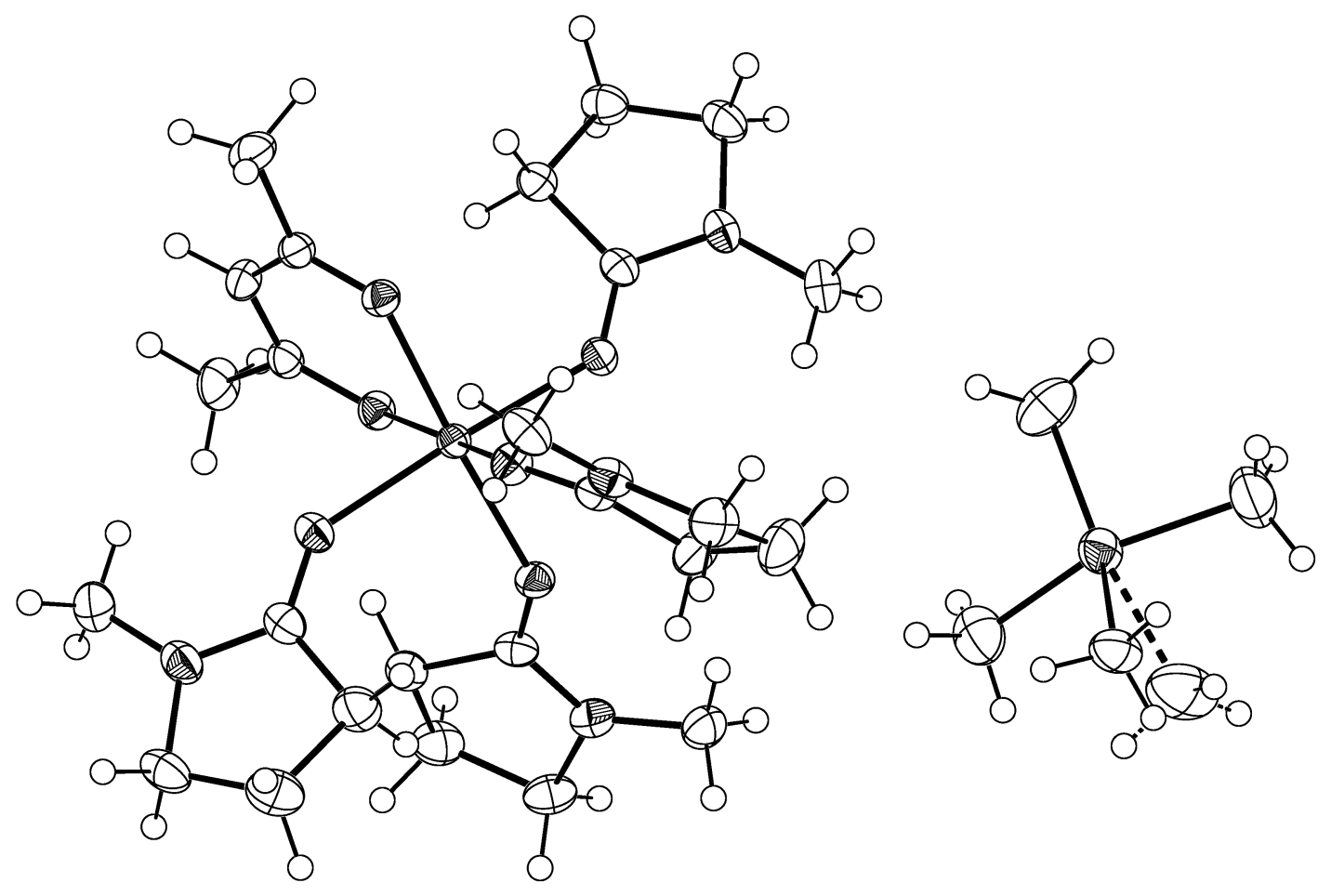


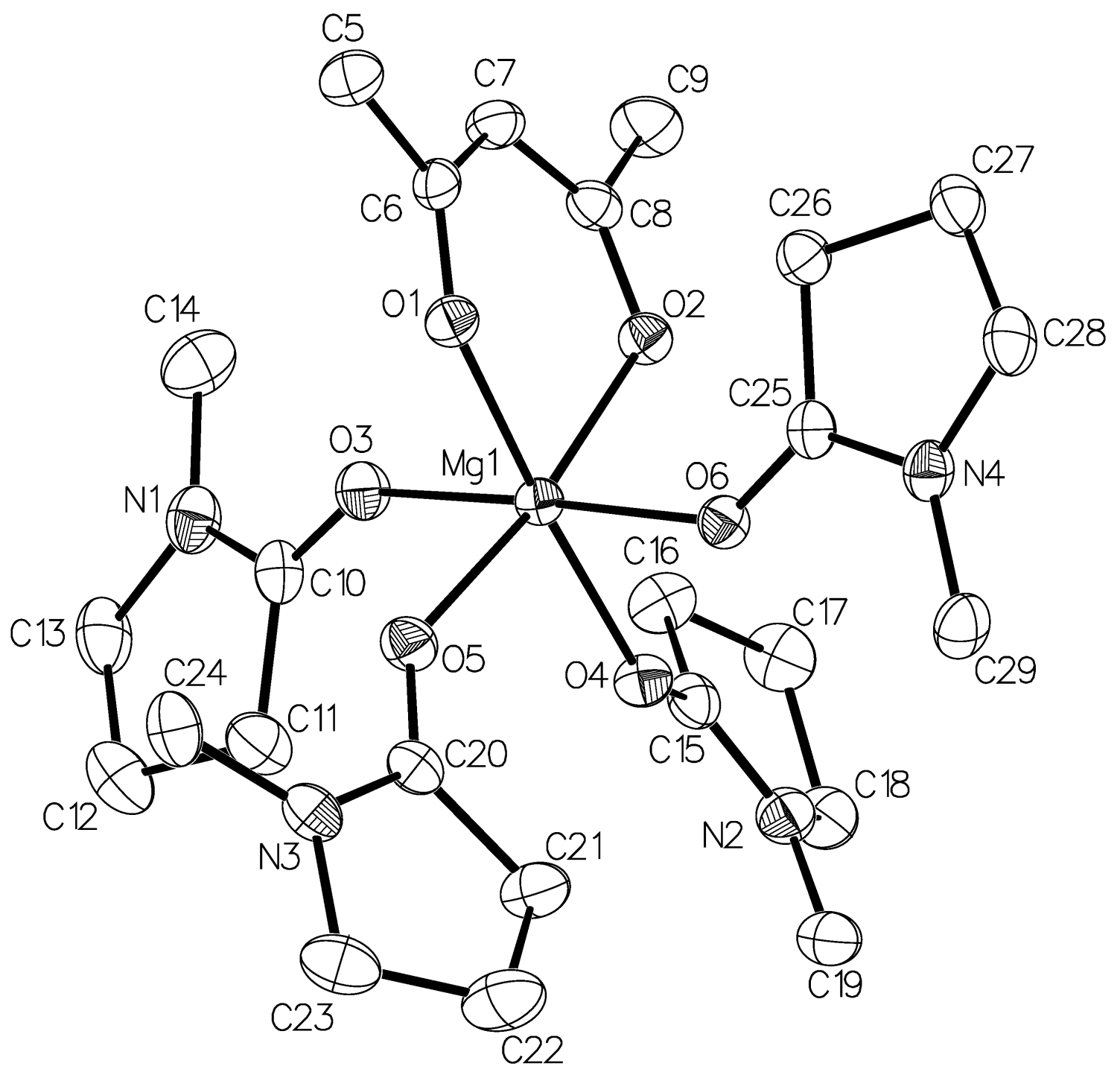



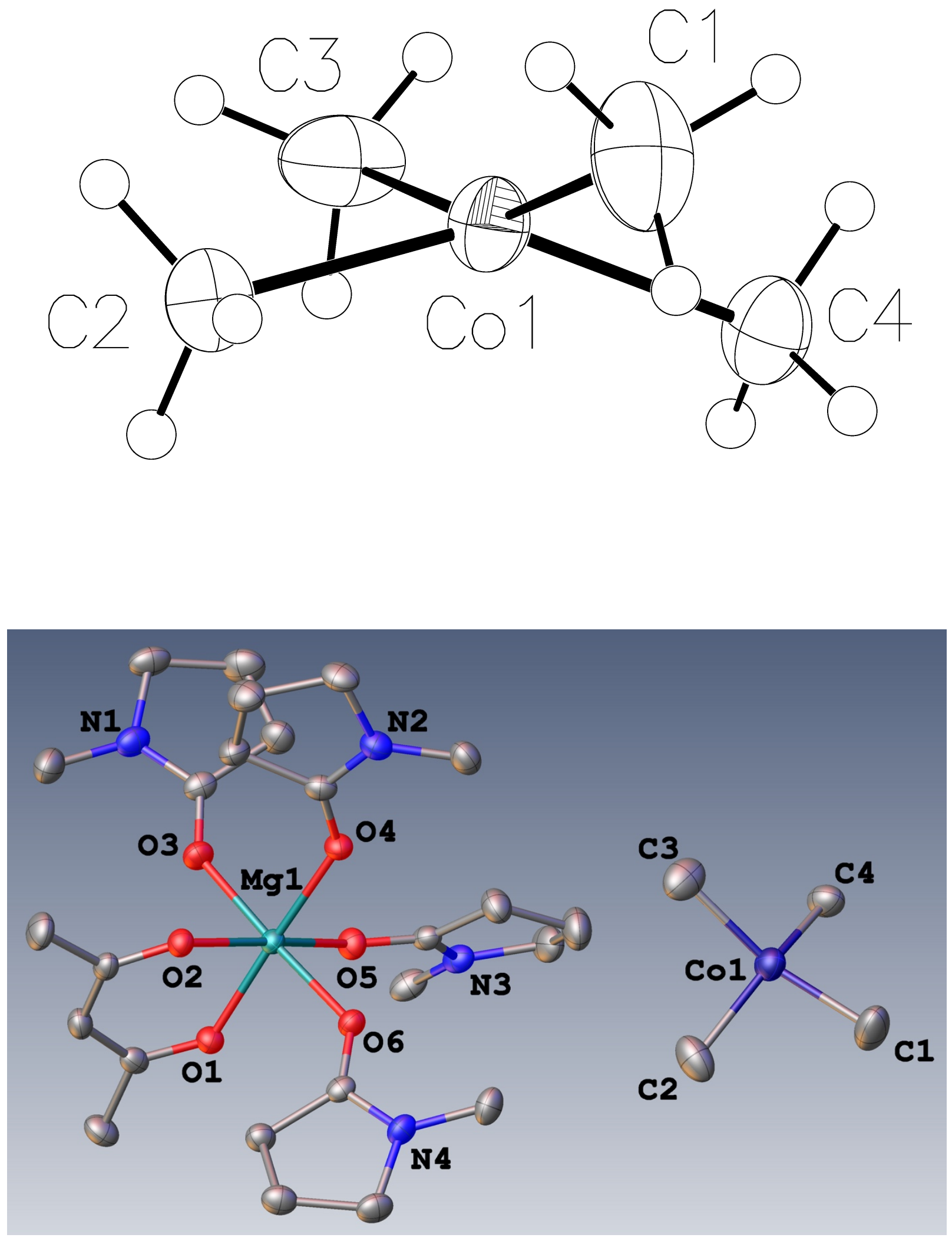
Table S6. Crystal data and structure refinement for neisc 68.

\begin{tabular}{|c|c|c|}
\hline Identification code & \multicolumn{2}{|l|}{ neisc68 } \\
\hline Empirical formula & \multicolumn{2}{|c|}{ C29 H55 Co Mg N4 O6 } \\
\hline Formula weight & \multicolumn{2}{|l|}{639.01} \\
\hline Temperature & \multicolumn{2}{|l|}{$100.01(10) \mathrm{K}$} \\
\hline Wavelength & \multicolumn{2}{|l|}{$1.54184 \AA$} \\
\hline Crystal system & \multicolumn{2}{|l|}{ triclinic } \\
\hline Space group & \multicolumn{2}{|l|}{$P-1$} \\
\hline \multirow[t]{3}{*}{ Unit cell dimensions } & $a=10.3493(2) \AA$ & $\alpha=83.2177(15)^{\circ}$ \\
\hline & $b=12.7559(2) \AA$ & $\beta=84.7733(16)^{\circ}$ \\
\hline & $c=13.1693(3) \AA$ & $\gamma=88.9855(16)^{\circ}$ \\
\hline Volume & \multicolumn{2}{|l|}{$1719.17(6) \AA^{3}$} \\
\hline$Z$ & \multicolumn{2}{|l|}{2} \\
\hline Density (calculated) & \multicolumn{2}{|l|}{$1.234 \mathrm{Mg} / \mathrm{m}^{3}$} \\
\hline Absorption coefficient & \multicolumn{2}{|l|}{$4.440 \mathrm{~mm}^{-1}$} \\
\hline$F(000)$ & \multicolumn{2}{|l|}{688} \\
\hline Crystal color, morphology & \multicolumn{2}{|l|}{ red, block } \\
\hline Crystal size & \multicolumn{2}{|c|}{$0.276 \times 0.189 \times 0.119 \mathrm{~mm}^{3}$} \\
\hline Theta range for data collection & \multicolumn{2}{|l|}{3.393 to $77.724^{\circ}$} \\
\hline Index ranges & \multicolumn{2}{|c|}{$-13 \leq h \leq 13,-16 \leq k \leq 14,-16 \leq l \leq 15$} \\
\hline Reflections collected & \multicolumn{2}{|l|}{25973} \\
\hline Independent reflections & \multicolumn{2}{|c|}{$7182[R($ int $)=0.0333]$} \\
\hline Observed reflections & \multicolumn{2}{|l|}{6867} \\
\hline Completeness to theta $=74.504^{\circ}$ & \multicolumn{2}{|l|}{$99.8 \%$} \\
\hline Absorption correction & \multicolumn{2}{|l|}{ Multi-scan } \\
\hline Max. and min. transmission & \multicolumn{2}{|c|}{1.00000 and 0.44846} \\
\hline Refinement method & \multicolumn{2}{|c|}{ Full-matrix least-squares on $F^{2}$} \\
\hline Data / restraints / parameters & \multicolumn{2}{|l|}{$7182 / 1 / 390$} \\
\hline Goodness-of-fit on $F^{2}$ & \multicolumn{2}{|l|}{1.094} \\
\hline Final $R$ indices $[I>2 \operatorname{sigma}(I)]$ & \multicolumn{2}{|c|}{$R 1=0.0437, w R 2=0.1259$} \\
\hline$R$ indices (all data) & \multicolumn{2}{|c|}{$R 1=0.0451, w R 2=0.1272$} \\
\hline Largest diff. peak and hole & \multicolumn{2}{|c|}{1.404 and -0.590 e. $\AA^{-3}$} \\
\hline
\end{tabular}


Table S7. Atomic coordinates $\left(\mathrm{x} 10^{4}\right)$ and equivalent isotropic displacement parameters $\left(\AA^{2} \times 10^{3}\right)$ for neisc68. $U_{e q}$ is defined as one third of the trace of the orthogonalized $U_{i j}$ tensor.

\begin{tabular}{|c|c|c|c|c|}
\hline & $\mathrm{x}$ & $\mathrm{y}$ & $\mathrm{z}$ & $\mathrm{U}_{\mathrm{eq}}$ \\
\hline Co1 & $9935(1)$ & $7837(1)$ & 2601(1) & $31(1)$ \\
\hline $\mathrm{C} 1$ & $10206(3)$ & $9366(2)$ & 2428(3) & $55(1)$ \\
\hline $\mathrm{C} 2$ & $8178(2)$ & $7977(2)$ & 2075(2) & $48(1)$ \\
\hline $\mathrm{C} 3$ & $10177(3)$ & $6387(2)$ & $2282(2)$ & $47(1)$ \\
\hline $\mathrm{C} 4$ & 10987(3) & $7629(2)$ & $3820(2)$ & $36(1)$ \\
\hline $\operatorname{Mg} 1$ & $5309(1)$ & 2835(1) & 2732(1) & $18(1)$ \\
\hline $\mathrm{O} 1$ & $3399(1)$ & 2913(1) & $3281(1)$ & $22(1)$ \\
\hline $\mathrm{O} 2$ & 4644(1) & 2196(1) & 1513(1) & $21(1)$ \\
\hline $\mathrm{O} 3$ & $5471(1)$ & 1293(1) & $3397(1)$ & $26(1)$ \\
\hline O4 & $7222(1)$ & 2832(1) & 2092(1) & $23(1)$ \\
\hline O5 & $5780(1)$ & $3353(1)$ & 4067(1) & $25(1)$ \\
\hline O6 & $5252(1)$ & 4391(1) & 2024(1) & $21(1)$ \\
\hline N1 & 6072(2) & $-372(1)$ & 3987(1) & $29(1)$ \\
\hline N2 & $9027(2)$ & 2590(1) & 1021(1) & $27(1)$ \\
\hline N3 & $6379(2)$ & $4254(1)$ & $5333(1)$ & $25(1)$ \\
\hline N4 & $4662(2)$ & $5915(1)$ & 1085(1) & $24(1)$ \\
\hline $\mathrm{C} 5$ & $1172(2)$ & $2519(2)$ & $3670(2)$ & $32(1)$ \\
\hline C6 & $2463(2)$ & 2391(1) & $3053(1)$ & $22(1)$ \\
\hline $\mathrm{C} 7$ & $2534(2)$ & $1728(1)$ & 2272(1) & $25(1)$ \\
\hline $\mathrm{C} 8$ & $3620(2)$ & $1657(1)$ & 1559(1) & $22(1)$ \\
\hline $\mathrm{C} 9$ & $3563(2)$ & $894(2)$ & $766(2)$ & $34(1)$ \\
\hline $\mathrm{C} 10$ & $6232(2)$ & $668(1)$ & $3832(1)$ & $24(1)$ \\
\hline $\mathrm{C} 11$ & $7486(2)$ & $936(2)$ & $4245(2)$ & $34(1)$ \\
\hline $\mathrm{C} 12$ & $7873(2)$ & $-83(2)$ & 4885(2) & $40(1)$ \\
\hline $\mathrm{C} 13$ & 7087(2) & $-955(2)$ & 4513(2) & $35(1)$ \\
\hline $\mathrm{C} 14$ & $5046(2)$ & $-905(2)$ & $3590(2)$ & $38(1)$ \\
\hline C15 & $7877(2)$ & $2302(1)$ & 1496(1) & $24(1)$ \\
\hline C16 & $7529(2)$ & $1236(1)$ & 1194(2) & $30(1)$ \\
\hline $\mathrm{C} 17$ & $8500(2)$ & $1086(2)$ & $280(2)$ & $35(1)$ \\
\hline $\mathrm{C} 18$ & $9628(2)$ & $1819(2)$ & $386(2)$ & $32(1)$ \\
\hline C19 & $9715(2)$ & $3517(2)$ & 1192(2) & $32(1)$ \\
\hline
\end{tabular}




\begin{tabular}{lrrrr}
$\mathrm{C} 20$ & $6520(2)$ & $3967(1)$ & $4388(1)$ & $22(1)$ \\
$\mathrm{C} 21$ & $7702(2)$ & $4493(2)$ & $3807(2)$ & $32(1)$ \\
$\mathrm{C} 22$ & $8073(2)$ & $5315(2)$ & $4493(2)$ & $44(1)$ \\
$\mathrm{C} 23$ & $7389(2)$ & $4956(2)$ & $5551(2)$ & $37(1)$ \\
$\mathrm{C} 24$ & $5413(2)$ & $3826(2)$ & $6125(1)$ & $31(1)$ \\
$\mathrm{C} 25$ & $4464(2)$ & $4918(1)$ & $1507(1)$ & $20(1)$ \\
$\mathrm{C} 26$ & $3145(2)$ & $4580(1)$ & $1275(1)$ & $24(1)$ \\
$\mathrm{C} 27$ & $2757(2)$ & $5428(2)$ & $430(1)$ & $28(1)$ \\
$\mathrm{C} 28$ & $3567(2)$ & $6386(1)$ & $562(2)$ & $29(1)$ \\
$\mathrm{C} 29$ & $5767(2)$ & $6537(1)$ & $1231(2)$ & $30(1)$ \\
$\mathrm{C} 4$ & $11823(4)$ & $7917(8)$ & $2834(8)$ & $64(3)$ \\
\hline
\end{tabular}


Table S8. Bond lengths $[\AA]$ and angles $\left[{ }^{\circ}\right]$ for neisc 68 .

\begin{tabular}{|c|c|c|c|}
\hline $\operatorname{Co}(1)-C(1)$ & $1.959(2)$ & $\mathrm{N}(2)-\mathrm{C}(19)$ & $1.441(2)$ \\
\hline $\mathrm{Co}(1)-\mathrm{C}(2)$ & $2.001(2)$ & $\mathrm{N}(3)-\mathrm{C}(20)$ & $1.333(2)$ \\
\hline $\operatorname{Co}(1)-C(3)$ & $1.952(2)$ & $\mathrm{N}(3)-\mathrm{C}(23)$ & $1.458(2)$ \\
\hline $\mathrm{Co}(1)-\mathrm{C}(4)$ & $2.010(3)$ & $\mathrm{N}(3)-\mathrm{C}(24)$ & $1.442(3)$ \\
\hline $\mathrm{Co}(1)-\mathrm{C}\left(4^{\prime}\right)$ & $2.012(3)$ & $\mathrm{N}(4)-\mathrm{C}(25)$ & $1.338(2)$ \\
\hline $\mathrm{C}(1)-\mathrm{H}(1 \mathrm{~A})$ & 0.9600 & $\mathrm{~N}(4)-\mathrm{C}(28)$ & $1.463(2)$ \\
\hline $\mathrm{C}(1)-\mathrm{H}(1 \mathrm{~B})$ & 0.9600 & N(4)-C(29) & $1.443(2)$ \\
\hline $\mathrm{C}(1)-\mathrm{H}(1 \mathrm{C})$ & 0.9600 & $\mathrm{C}(5)-\mathrm{H}(5 \mathrm{~A})$ & 0.9600 \\
\hline $\mathrm{C}(2)-\mathrm{H}(2 \mathrm{~A})$ & 0.9600 & $\mathrm{C}(5)-\mathrm{H}(5 \mathrm{~B})$ & 0.9600 \\
\hline $\mathrm{C}(2)-\mathrm{H}(2 \mathrm{~B})$ & 0.9600 & $\mathrm{C}(5)-\mathrm{H}(5 \mathrm{C})$ & 0.9600 \\
\hline $\mathrm{C}(2)-\mathrm{H}(2 \mathrm{C})$ & 0.9600 & $C(5)-C(6)$ & $1.516(2)$ \\
\hline $\mathrm{C}(3)-\mathrm{H}(3 \mathrm{~A})$ & 0.9600 & $C(6)-C(7)$ & $1.402(3)$ \\
\hline $\mathrm{C}(3)-\mathrm{H}(3 \mathrm{~B})$ & 0.9600 & $\mathrm{C}(7)-\mathrm{H}(7)$ & 0.9300 \\
\hline $\mathrm{C}(3)-\mathrm{H}(3 \mathrm{C})$ & 0.9600 & $C(7)-C(8)$ & $1.407(3)$ \\
\hline $\mathrm{C}(4)-\mathrm{H}(4 \mathrm{~A})$ & 0.9600 & $\mathrm{C}(8)-\mathrm{C}(9)$ & $1.514(2)$ \\
\hline $\mathrm{C}(4)-\mathrm{H}(4 \mathrm{~B})$ & 0.9600 & $\mathrm{C}(9)-\mathrm{H}(9 \mathrm{~A})$ & 0.9600 \\
\hline $\mathrm{C}(4)-\mathrm{H}(4 \mathrm{C})$ & 0.9600 & $\mathrm{C}(9)-\mathrm{H}(9 \mathrm{~B})$ & 0.9600 \\
\hline $\mathrm{Mg}(1)-\mathrm{O}(1)$ & $2.0466(13)$ & $\mathrm{C}(9)-\mathrm{H}(9 \mathrm{C})$ & 0.9600 \\
\hline $\mathrm{Mg}(1)-\mathrm{O}(2)$ & $2.0605(13)$ & $C(10)-C(11)$ & $1.512(3)$ \\
\hline $\mathrm{Mg}(1)-\mathrm{O}(3)$ & $2.0666(13)$ & $\mathrm{C}(11)-\mathrm{H}(11 \mathrm{~A})$ & 0.9700 \\
\hline $\mathrm{Mg}(1)-\mathrm{O}(4)$ & $2.0799(13)$ & $\mathrm{C}(11)-\mathrm{H}(11 \mathrm{~B})$ & 0.9700 \\
\hline $\mathrm{Mg}(1)-\mathrm{O}(5)$ & $2.0509(13)$ & $C(11)-C(12)$ & $1.532(3)$ \\
\hline $\mathrm{Mg}(1)-\mathrm{O}(6)$ & $2.0936(12)$ & $\mathrm{C}(12)-\mathrm{H}(12 \mathrm{~A})$ & 0.9700 \\
\hline $\mathrm{O}(1)-\mathrm{C}(6)$ & $1.263(2)$ & $\mathrm{C}(12)-\mathrm{H}(12 \mathrm{~B})$ & 0.9700 \\
\hline $\mathrm{O}(2)-\mathrm{C}(8)$ & $1.265(2)$ & $C(12)-C(13)$ & $1.540(3)$ \\
\hline $\mathrm{O}(3)-\mathrm{C}(10)$ & $1.239(2)$ & $\mathrm{C}(13)-\mathrm{H}(13 \mathrm{~A})$ & 0.9700 \\
\hline $\mathrm{O}(4)-\mathrm{C}(15)$ & $1.243(2)$ & $\mathrm{C}(13)-\mathrm{H}(13 \mathrm{~B})$ & 0.9700 \\
\hline $\mathrm{O}(5)-\mathrm{C}(20)$ & $1.239(2)$ & $\mathrm{C}(14)-\mathrm{H}(14 \mathrm{~A})$ & 0.9600 \\
\hline $\mathrm{O}(6)-\mathrm{C}(25)$ & $1.246(2)$ & $\mathrm{C}(14)-\mathrm{H}(14 \mathrm{~B})$ & 0.9600 \\
\hline $\mathrm{N}(1)-\mathrm{C}(10)$ & $1.329(2)$ & $\mathrm{C}(14)-\mathrm{H}(14 \mathrm{C})$ & 0.9600 \\
\hline $\mathrm{N}(1)-\mathrm{C}(13)$ & $1.456(3)$ & $C(15)-C(16)$ & $1.519(2)$ \\
\hline $\mathrm{N}(1)-\mathrm{C}(14)$ & $1.440(3)$ & $C(16)-H(16 A)$ & 0.9700 \\
\hline $\mathrm{N}(2)-\mathrm{C}(15)$ & $1.330(2)$ & $\mathrm{C}(16)-\mathrm{H}(16 \mathrm{~B})$ & 0.9700 \\
\hline $\mathrm{N}(2)-\mathrm{C}(18)$ & $1.460(2)$ & $C(16)-C(17)$ & $1.524(3)$ \\
\hline
\end{tabular}




\begin{tabular}{|c|c|c|c|}
\hline $\mathrm{C}(17)-\mathrm{H}(17 \mathrm{~A})$ & 0.9700 & $\mathrm{C}(1)-\mathrm{Co}(1)-\mathrm{C}(4)$ & $92.13(13)$ \\
\hline $\mathrm{C}(17)-\mathrm{H}(17 \mathrm{~B})$ & 0.9700 & $\mathrm{C}(1)-\mathrm{Co}(1)-\mathrm{C}\left(4^{\prime}\right)$ & $78.6(3)$ \\
\hline$C(17)-C(18)$ & $1.535(3)$ & $\mathrm{C}(2)-\mathrm{Co}(1)-\mathrm{C}(4)$ & $147.85(12)$ \\
\hline $\mathrm{C}(18)-\mathrm{H}(18 \mathrm{~A})$ & 0.9700 & $\mathrm{C}(2)-\mathrm{Co}(1)-\mathrm{C}\left(4^{\prime}\right)$ & $166.8(3)$ \\
\hline $\mathrm{C}(18)-\mathrm{H}(18 \mathrm{~B})$ & 0.9700 & $\mathrm{C}(3)-\mathrm{Co}(1)-\mathrm{C}(1)$ & $156.46(13)$ \\
\hline $\mathrm{C}(19)-\mathrm{H}(19 \mathrm{~A})$ & 0.9600 & $\mathrm{C}(3)-\mathrm{Co}(1)-\mathrm{C}(2)$ & $93.52(12)$ \\
\hline C(19)-H(19B) & 0.9600 & $\mathrm{C}(3)-\mathrm{Co}(1)-\mathrm{C}(4)$ & $94.21(13)$ \\
\hline C(19)-H(19C) & 0.9600 & $\mathrm{C}(3)-\mathrm{Co}(1)-\mathrm{C}\left(4^{\prime}\right)$ & $90.4(3)$ \\
\hline$C(20)-C(21)$ & $1.508(3)$ & $\mathrm{Co}(1)-\mathrm{C}(1)-\mathrm{H}(1 \mathrm{~A})$ & 109.5 \\
\hline $\mathrm{C}(21)-\mathrm{H}(21 \mathrm{~A})$ & 0.9700 & $\mathrm{Co}(1)-\mathrm{C}(1)-\mathrm{H}(1 \mathrm{~B})$ & 109.5 \\
\hline $\mathrm{C}(21)-\mathrm{H}(21 \mathrm{~B})$ & 0.9700 & $\mathrm{Co}(1)-\mathrm{C}(1)-\mathrm{H}(1 \mathrm{C})$ & 109.5 \\
\hline $\mathrm{C}(21)-\mathrm{C}(22)$ & $1.537(3)$ & $\mathrm{H}(1 \mathrm{~A})-\mathrm{C}(1)-\mathrm{H}(1 \mathrm{~B})$ & 109.5 \\
\hline $\mathrm{C}(22)-\mathrm{H}(22 \mathrm{~A})$ & 0.9700 & $\mathrm{H}(1 \mathrm{~A})-\mathrm{C}(1)-\mathrm{H}(1 \mathrm{C})$ & 109.5 \\
\hline $\mathrm{C}(22)-\mathrm{H}(22 \mathrm{~B})$ & 0.9700 & $\mathrm{H}(1 \mathrm{~B})-\mathrm{C}(1)-\mathrm{H}(1 \mathrm{C})$ & 109.5 \\
\hline$C(22)-C(23)$ & $1.527(3)$ & $\mathrm{Co}(1)-\mathrm{C}(2)-\mathrm{H}(2 \mathrm{~A})$ & 109.5 \\
\hline $\mathrm{C}(23)-\mathrm{H}(23 \mathrm{~A})$ & 0.9700 & $\mathrm{Co}(1)-\mathrm{C}(2)-\mathrm{H}(2 \mathrm{~B})$ & 109.5 \\
\hline $\mathrm{C}(23)-\mathrm{H}(23 \mathrm{~B})$ & 0.9700 & $\mathrm{Co}(1)-\mathrm{C}(2)-\mathrm{H}(2 \mathrm{C})$ & 109.5 \\
\hline $\mathrm{C}(24)-\mathrm{H}(24 \mathrm{~A})$ & 0.9600 & $\mathrm{H}(2 \mathrm{~A})-\mathrm{C}(2)-\mathrm{H}(2 \mathrm{~B})$ & 109.5 \\
\hline $\mathrm{C}(24)-\mathrm{H}(24 \mathrm{~B})$ & 0.9600 & $\mathrm{H}(2 \mathrm{~A})-\mathrm{C}(2)-\mathrm{H}(2 \mathrm{C})$ & 109.5 \\
\hline $\mathrm{C}(24)-\mathrm{H}(24 \mathrm{C})$ & 0.9600 & $\mathrm{H}(2 \mathrm{~B})-\mathrm{C}(2)-\mathrm{H}(2 \mathrm{C})$ & 109.5 \\
\hline$C(25)-C(26)$ & $1.506(2)$ & $\mathrm{Co}(1)-\mathrm{C}(3)-\mathrm{H}(3 \mathrm{~A})$ & 109.5 \\
\hline $\mathrm{C}(26)-\mathrm{H}(26 \mathrm{~A})$ & 0.9700 & $\mathrm{Co}(1)-\mathrm{C}(3)-\mathrm{H}(3 \mathrm{~B})$ & 109.5 \\
\hline $\mathrm{C}(26)-\mathrm{H}(26 \mathrm{~B})$ & 0.9700 & $\mathrm{Co}(1)-\mathrm{C}(3)-\mathrm{H}(3 \mathrm{C})$ & 109.5 \\
\hline$C(26)-C(27)$ & $1.534(2)$ & $\mathrm{H}(3 \mathrm{~A})-\mathrm{C}(3)-\mathrm{H}(3 \mathrm{~B})$ & 109.5 \\
\hline $\mathrm{C}(27)-\mathrm{H}(27 \mathrm{~A})$ & 0.9700 & $\mathrm{H}(3 \mathrm{~A})-\mathrm{C}(3)-\mathrm{H}(3 \mathrm{C})$ & 109.5 \\
\hline $\mathrm{C}(27)-\mathrm{H}(27 \mathrm{~B})$ & 0.9700 & $\mathrm{H}(3 \mathrm{~B})-\mathrm{C}(3)-\mathrm{H}(3 \mathrm{C})$ & 109.5 \\
\hline $\mathrm{C}(27)-\mathrm{C}(28)$ & $1.530(3)$ & $\mathrm{Co}(1)-\mathrm{C}(4)-\mathrm{H}(4 \mathrm{~A})$ & 109.5 \\
\hline $\mathrm{C}(28)-\mathrm{H}(28 \mathrm{~A})$ & 0.9700 & $\mathrm{Co}(1)-\mathrm{C}(4)-\mathrm{H}(4 \mathrm{~B})$ & 109.5 \\
\hline $\mathrm{C}(28)-\mathrm{H}(28 \mathrm{~B})$ & 0.9700 & $\mathrm{Co}(1)-\mathrm{C}(4)-\mathrm{H}(4 \mathrm{C})$ & 109.5 \\
\hline $\mathrm{C}(29)-\mathrm{H}(29 \mathrm{~A})$ & 0.9600 & $\mathrm{H}(4 \mathrm{~A})-\mathrm{C}(4)-\mathrm{H}(4 \mathrm{~B})$ & 109.5 \\
\hline $\mathrm{C}(29)-\mathrm{H}(29 \mathrm{~B})$ & 0.9600 & $\mathrm{H}(4 \mathrm{~A})-\mathrm{C}(4)-\mathrm{H}(4 \mathrm{C})$ & 109.5 \\
\hline $\mathrm{C}(29)-\mathrm{H}(29 \mathrm{C})$ & 0.9600 & $\mathrm{H}(4 \mathrm{~B})-\mathrm{C}(4)-\mathrm{H}(4 \mathrm{C})$ & 109.5 \\
\hline $\mathrm{C}\left(4^{\prime}\right)-\mathrm{H}\left(4^{\prime} \mathrm{A}\right)$ & 0.9602 & $\mathrm{O}(1)-\mathrm{Mg}(1)-\mathrm{O}(2)$ & $85.92(5)$ \\
\hline $\mathrm{C}\left(4^{\prime}\right)-\mathrm{H}\left(4^{\prime} \mathrm{B}\right)$ & 0.9602 & $\mathrm{O}(1)-\mathrm{Mg}(1)-\mathrm{O}(3)$ & $91.77(5)$ \\
\hline $\mathrm{C}\left(4^{\prime}\right)-\mathrm{H}\left(4^{\prime} \mathrm{C}\right)$ & 0.9601 & $\mathrm{O}(1)-\mathrm{Mg}(1)-\mathrm{O}(4)$ & $176.10(6)$ \\
\hline $\mathrm{C}(1)-\mathrm{Co}(1)-\mathrm{C}(2)$ & $93.09(12)$ & $\mathrm{O}(1)-\mathrm{Mg}(1)-\mathrm{O}(5)$ & $88.03(5)$ \\
\hline
\end{tabular}




\begin{tabular}{|c|c|c|c|}
\hline $\mathrm{O}(1)-\mathrm{Mg}(1)-\mathrm{O}(6)$ & $91.41(5)$ & $\mathrm{O}(1)-\mathrm{C}(6)-\mathrm{C}(7)$ & $124.96(15)$ \\
\hline $\mathrm{O}(2)-\mathrm{Mg}(1)-\mathrm{O}(3)$ & $85.81(5)$ & $C(7)-C(6)-C(5)$ & $118.69(16)$ \\
\hline $\mathrm{O}(2)-\mathrm{Mg}(1)-\mathrm{O}(4)$ & $92.55(5)$ & $\mathrm{C}(6)-\mathrm{C}(7)-\mathrm{H}(7)$ & 118.1 \\
\hline $\mathrm{O}(2)-\mathrm{Mg}(1)-\mathrm{O}(6)$ & $94.27(5)$ & $C(6)-C(7)-C(8)$ & $123.90(16)$ \\
\hline $\mathrm{O}(3)-\mathrm{Mg}(1)-\mathrm{O}(4)$ & $91.69(5)$ & $\mathrm{C}(8)-\mathrm{C}(7)-\mathrm{H}(7)$ & 118.1 \\
\hline $\mathrm{O}(3)-\mathrm{Mg}(1)-\mathrm{O}(6)$ & $176.81(6)$ & $\mathrm{O}(2)-\mathrm{C}(8)-\mathrm{C}(7)$ & $125.15(15)$ \\
\hline $\mathrm{O}(4)-\mathrm{Mg}(1)-\mathrm{O}(6)$ & $85.12(5)$ & $\mathrm{O}(2)-\mathrm{C}(8)-\mathrm{C}(9)$ & $116.87(16)$ \\
\hline $\mathrm{O}(5)-\mathrm{Mg}(1)-\mathrm{O}(2)$ & $172.26(6)$ & $\mathrm{C}(7)-\mathrm{C}(8)-\mathrm{C}(9)$ & $117.97(16)$ \\
\hline $\mathrm{O}(5)-\mathrm{Mg}(1)-\mathrm{O}(3)$ & $89.57(5)$ & $\mathrm{C}(8)-\mathrm{C}(9)-\mathrm{H}(9 \mathrm{~A})$ & 109.5 \\
\hline $\mathrm{O}(5)-\mathrm{Mg}(1)-\mathrm{O}(4)$ & $93.79(5)$ & $\mathrm{C}(8)-\mathrm{C}(9)-\mathrm{H}(9 \mathrm{~B})$ & 109.5 \\
\hline $\mathrm{O}(5)-\mathrm{Mg}(1)-\mathrm{O}(6)$ & $90.69(5)$ & $\mathrm{C}(8)-\mathrm{C}(9)-\mathrm{H}(9 \mathrm{C})$ & 109.5 \\
\hline $\mathrm{C}(6)-\mathrm{O}(1)-\mathrm{Mg}(1)$ & $127.71(11)$ & $\mathrm{H}(9 \mathrm{~A})-\mathrm{C}(9)-\mathrm{H}(9 \mathrm{~B})$ & 109.5 \\
\hline $\mathrm{C}(8)-\mathrm{O}(2)-\mathrm{Mg}(1)$ & $124.90(11)$ & $\mathrm{H}(9 \mathrm{~A})-\mathrm{C}(9)-\mathrm{H}(9 \mathrm{C})$ & 109.5 \\
\hline $\mathrm{C}(10)-\mathrm{O}(3)-\mathrm{Mg}(1)$ & $142.37(12)$ & $\mathrm{H}(9 \mathrm{~B})-\mathrm{C}(9)-\mathrm{H}(9 \mathrm{C})$ & 109.5 \\
\hline $\mathrm{C}(15)-\mathrm{O}(4)-\mathrm{Mg}(1)$ & $135.90(12)$ & $\mathrm{O}(3)-\mathrm{C}(10)-\mathrm{N}(1)$ & $124.18(18)$ \\
\hline $\mathrm{C}(20)-\mathrm{O}(5)-\mathrm{Mg}(1)$ & $141.44(12)$ & $\mathrm{O}(3)-\mathrm{C}(10)-\mathrm{C}(11)$ & $126.92(17)$ \\
\hline $\mathrm{C}(25)-\mathrm{O}(6)-\mathrm{Mg}(1)$ & $134.46(11)$ & $\mathrm{N}(1)-\mathrm{C}(10)-\mathrm{C}(11)$ & $108.88(16)$ \\
\hline$C(10)-N(1)-C(13)$ & $114.87(17)$ & $\mathrm{C}(10)-\mathrm{C}(11)-\mathrm{H}(11 \mathrm{~A})$ & 110.9 \\
\hline$C(10)-N(1)-C(14)$ & $123.31(17)$ & $\mathrm{C}(10)-\mathrm{C}(11)-\mathrm{H}(11 \mathrm{~B})$ & 110.9 \\
\hline $\mathrm{C}(14)-\mathrm{N}(1)-\mathrm{C}(13)$ & $121.59(16)$ & $\mathrm{C}(10)-\mathrm{C}(11)-\mathrm{C}(12)$ & $104.41(17)$ \\
\hline$C(15)-N(2)-C(18)$ & $114.19(16)$ & $\mathrm{H}(11 \mathrm{~A})-\mathrm{C}(11)-\mathrm{H}(11 \mathrm{~B})$ & 108.9 \\
\hline$C(15)-N(2)-C(19)$ & $124.17(16)$ & $\mathrm{C}(12)-\mathrm{C}(11)-\mathrm{H}(11 \mathrm{~A})$ & 110.9 \\
\hline $\mathrm{C}(19)-\mathrm{N}(2)-\mathrm{C}(18)$ & $121.27(16)$ & $\mathrm{C}(12)-\mathrm{C}(11)-\mathrm{H}(11 \mathrm{~B})$ & 110.9 \\
\hline $\mathrm{C}(20)-\mathrm{N}(3)-\mathrm{C}(23)$ & $114.16(16)$ & $\mathrm{C}(11)-\mathrm{C}(12)-\mathrm{H}(12 \mathrm{~A})$ & 110.8 \\
\hline $\mathrm{C}(20)-\mathrm{N}(3)-\mathrm{C}(24)$ & $124.06(16)$ & $\mathrm{C}(11)-\mathrm{C}(12)-\mathrm{H}(12 \mathrm{~B})$ & 110.8 \\
\hline$C(24)-N(3)-C(23)$ & $121.41(16)$ & $\mathrm{C}(11)-\mathrm{C}(12)-\mathrm{C}(13)$ & $104.77(16)$ \\
\hline $\mathrm{C}(25)-\mathrm{N}(4)-\mathrm{C}(28)$ & $113.89(15)$ & $\mathrm{H}(12 \mathrm{~A})-\mathrm{C}(12)-\mathrm{H}(12 \mathrm{~B})$ & 108.9 \\
\hline $\mathrm{C}(25)-\mathrm{N}(4)-\mathrm{C}(29)$ & $124.31(16)$ & $\mathrm{C}(13)-\mathrm{C}(12)-\mathrm{H}(12 \mathrm{~A})$ & 110.8 \\
\hline $\mathrm{C}(29)-\mathrm{N}(4)-\mathrm{C}(28)$ & $121.31(15)$ & $\mathrm{C}(13)-\mathrm{C}(12)-\mathrm{H}(12 \mathrm{~B})$ & 110.8 \\
\hline $\mathrm{H}(5 \mathrm{~A})-\mathrm{C}(5)-\mathrm{H}(5 \mathrm{~B})$ & 109.5 & $\mathrm{~N}(1)-\mathrm{C}(13)-\mathrm{C}(12)$ & $103.52(16)$ \\
\hline $\mathrm{H}(5 \mathrm{~A})-\mathrm{C}(5)-\mathrm{H}(5 \mathrm{C})$ & 109.5 & $\mathrm{~N}(1)-\mathrm{C}(13)-\mathrm{H}(13 \mathrm{~A})$ & 111.1 \\
\hline $\mathrm{H}(5 \mathrm{~B})-\mathrm{C}(5)-\mathrm{H}(5 \mathrm{C})$ & 109.5 & $\mathrm{~N}(1)-\mathrm{C}(13)-\mathrm{H}(13 \mathrm{~B})$ & 111.1 \\
\hline $\mathrm{C}(6)-\mathrm{C}(5)-\mathrm{H}(5 \mathrm{~A})$ & 109.5 & $\mathrm{C}(12)-\mathrm{C}(13)-\mathrm{H}(13 \mathrm{~A})$ & 111.1 \\
\hline $\mathrm{C}(6)-\mathrm{C}(5)-\mathrm{H}(5 \mathrm{~B})$ & 109.5 & $\mathrm{C}(12)-\mathrm{C}(13)-\mathrm{H}(13 \mathrm{~B})$ & 111.1 \\
\hline $\mathrm{C}(6)-\mathrm{C}(5)-\mathrm{H}(5 \mathrm{C})$ & 109.5 & $\mathrm{H}(13 \mathrm{~A})-\mathrm{C}(13)-\mathrm{H}(13 \mathrm{~B})$ & 109.0 \\
\hline $\mathrm{O}(1)-\mathrm{C}(6)-\mathrm{C}(5)$ & $116.34(16)$ & $\mathrm{N}(1)-\mathrm{C}(14)-\mathrm{H}(14 \mathrm{~A})$ & 109.5 \\
\hline
\end{tabular}




\begin{tabular}{|c|c|c|c|}
\hline N(1)-C(14)-H(14B) & 109.5 & $\mathrm{C}(20)-\mathrm{C}(21)-\mathrm{H}(21 \mathrm{~B})$ & 111.0 \\
\hline $\mathrm{N}(1)-\mathrm{C}(14)-\mathrm{H}(14 \mathrm{C})$ & 109.5 & $\mathrm{C}(20)-\mathrm{C}(21)-\mathrm{C}(22)$ & $103.98(16)$ \\
\hline $\mathrm{H}(14 \mathrm{~A})-\mathrm{C}(14)-\mathrm{H}(14 \mathrm{~B})$ & 109.5 & $H(21 A)-C(21)-H(21 B)$ & 109.0 \\
\hline $\mathrm{H}(14 \mathrm{~A})-\mathrm{C}(14)-\mathrm{H}(14 \mathrm{C})$ & 109.5 & $\mathrm{C}(22)-\mathrm{C}(21)-\mathrm{H}(21 \mathrm{~A})$ & 111.0 \\
\hline $\mathrm{H}(14 \mathrm{~B})-\mathrm{C}(14)-\mathrm{H}(14 \mathrm{C})$ & 109.5 & $\mathrm{C}(22)-\mathrm{C}(21)-\mathrm{H}(21 \mathrm{~B})$ & 111.0 \\
\hline $\mathrm{O}(4)-\mathrm{C}(15)-\mathrm{N}(2)$ & $124.34(17)$ & $\mathrm{C}(21)-\mathrm{C}(22)-\mathrm{H}(22 \mathrm{~A})$ & 110.8 \\
\hline $\mathrm{O}(4)-\mathrm{C}(15)-\mathrm{C}(16)$ & $126.93(16)$ & $\mathrm{C}(21)-\mathrm{C}(22)-\mathrm{H}(22 \mathrm{~B})$ & 110.8 \\
\hline $\mathrm{N}(2)-\mathrm{C}(15)-\mathrm{C}(16)$ & $108.72(15)$ & $\mathrm{H}(22 \mathrm{~A})-\mathrm{C}(22)-\mathrm{H}(22 \mathrm{~B})$ & 108.8 \\
\hline $\mathrm{C}(15)-\mathrm{C}(16)-\mathrm{H}(16 \mathrm{~A})$ & 110.9 & $\mathrm{C}(23)-\mathrm{C}(22)-\mathrm{C}(21)$ & 104.93(17) \\
\hline $\mathrm{C}(15)-\mathrm{C}(16)-\mathrm{H}(16 \mathrm{~B})$ & 110.9 & $\mathrm{C}(23)-\mathrm{C}(22)-\mathrm{H}(22 \mathrm{~A})$ & 110.8 \\
\hline$C(15)-C(16)-C(17)$ & $104.05(15)$ & $\mathrm{C}(23)-\mathrm{C}(22)-\mathrm{H}(22 \mathrm{~B})$ & 110.8 \\
\hline $\mathrm{H}(16 \mathrm{~A})-\mathrm{C}(16)-\mathrm{H}(16 \mathrm{~B})$ & 109.0 & $\mathrm{~N}(3)-\mathrm{C}(23)-\mathrm{C}(22)$ & $103.65(16)$ \\
\hline $\mathrm{C}(17)-\mathrm{C}(16)-\mathrm{H}(16 \mathrm{~A})$ & 110.9 & $\mathrm{~N}(3)-\mathrm{C}(23)-\mathrm{H}(23 \mathrm{~A})$ & 111.0 \\
\hline $\mathrm{C}(17)-\mathrm{C}(16)-\mathrm{H}(16 \mathrm{~B})$ & 110.9 & $\mathrm{~N}(3)-\mathrm{C}(23)-\mathrm{H}(23 \mathrm{~B})$ & 111.0 \\
\hline$C(16)-C(17)-H(17 A)$ & 110.8 & $\mathrm{C}(22)-\mathrm{C}(23)-\mathrm{H}(23 \mathrm{~A})$ & 111.0 \\
\hline $\mathrm{C}(16)-\mathrm{C}(17)-\mathrm{H}(17 \mathrm{~B})$ & 110.8 & $\mathrm{C}(22)-\mathrm{C}(23)-\mathrm{H}(23 \mathrm{~B})$ & 111.0 \\
\hline $\mathrm{C}(16)-\mathrm{C}(17)-\mathrm{C}(18)$ & $104.52(15)$ & $\mathrm{H}(23 \mathrm{~A})-\mathrm{C}(23)-\mathrm{H}(23 \mathrm{~B})$ & 109.0 \\
\hline $\mathrm{H}(17 \mathrm{~A})-\mathrm{C}(17)-\mathrm{H}(17 \mathrm{~B})$ & 108.9 & $\mathrm{~N}(3)-\mathrm{C}(24)-\mathrm{H}(24 \mathrm{~A})$ & 109.5 \\
\hline $\mathrm{C}(18)-\mathrm{C}(17)-\mathrm{H}(17 \mathrm{~A})$ & 110.8 & $\mathrm{~N}(3)-\mathrm{C}(24)-\mathrm{H}(24 \mathrm{~B})$ & 109.5 \\
\hline $\mathrm{C}(18)-\mathrm{C}(17)-\mathrm{H}(17 \mathrm{~B})$ & 110.8 & $\mathrm{~N}(3)-\mathrm{C}(24)-\mathrm{H}(24 \mathrm{C})$ & 109.5 \\
\hline $\mathrm{N}(2)-\mathrm{C}(18)-\mathrm{C}(17)$ & $103.16(15)$ & $\mathrm{H}(24 \mathrm{~A})-\mathrm{C}(24)-\mathrm{H}(24 \mathrm{~B})$ & 109.5 \\
\hline $\mathrm{N}(2)-\mathrm{C}(18)-\mathrm{H}(18 \mathrm{~A})$ & 111.1 & $\mathrm{H}(24 \mathrm{~A})-\mathrm{C}(24)-\mathrm{H}(24 \mathrm{C})$ & 109.5 \\
\hline $\mathrm{N}(2)-\mathrm{C}(18)-\mathrm{H}(18 \mathrm{~B})$ & 111.1 & $\mathrm{H}(24 \mathrm{~B})-\mathrm{C}(24)-\mathrm{H}(24 \mathrm{C})$ & 109.5 \\
\hline $\mathrm{C}(17)-\mathrm{C}(18)-\mathrm{H}(18 \mathrm{~A})$ & 111.1 & $\mathrm{O}(6)-\mathrm{C}(25)-\mathrm{N}(4)$ & $123.97(16)$ \\
\hline $\mathrm{C}(17)-\mathrm{C}(18)-\mathrm{H}(18 \mathrm{~B})$ & 111.1 & $\mathrm{O}(6)-\mathrm{C}(25)-\mathrm{C}(26)$ & $127.32(15)$ \\
\hline $\mathrm{H}(18 \mathrm{~A})-\mathrm{C}(18)-\mathrm{H}(18 \mathrm{~B})$ & 109.1 & $\mathrm{~N}(4)-\mathrm{C}(25)-\mathrm{C}(26)$ & $108.69(15)$ \\
\hline N(2)-C(19)-H(19A) & 109.5 & $C(25)-C(26)-H(26 A)$ & 110.9 \\
\hline N(2)-C(19)-H(19B) & 109.5 & $\mathrm{C}(25)-\mathrm{C}(26)-\mathrm{H}(26 \mathrm{~B})$ & 110.9 \\
\hline $\mathrm{N}(2)-\mathrm{C}(19)-\mathrm{H}(19 \mathrm{C})$ & 109.5 & $C(25)-C(26)-C(27)$ & $104.16(14)$ \\
\hline $\mathrm{H}(19 \mathrm{~A})-\mathrm{C}(19)-\mathrm{H}(19 \mathrm{~B})$ & 109.5 & $\mathrm{H}(26 \mathrm{~A})-\mathrm{C}(26)-\mathrm{H}(26 \mathrm{~B})$ & 108.9 \\
\hline $\mathrm{H}(19 \mathrm{~A})-\mathrm{C}(19)-\mathrm{H}(19 \mathrm{C})$ & 109.5 & $\mathrm{C}(27)-\mathrm{C}(26)-\mathrm{H}(26 \mathrm{~A})$ & 110.9 \\
\hline H(19B)-C(19)-H(19C) & 109.5 & $C(27)-C(26)-H(26 B)$ & 110.9 \\
\hline $\mathrm{O}(5)-\mathrm{C}(20)-\mathrm{N}(3)$ & $123.82(17)$ & $\mathrm{C}(26)-\mathrm{C}(27)-\mathrm{H}(27 \mathrm{~A})$ & 110.9 \\
\hline $\mathrm{O}(5)-\mathrm{C}(20)-\mathrm{C}(21)$ & $126.88(16)$ & $\mathrm{C}(26)-\mathrm{C}(27)-\mathrm{H}(27 \mathrm{~B})$ & 110.9 \\
\hline $\mathrm{N}(3)-\mathrm{C}(20)-\mathrm{C}(21)$ & $109.29(15)$ & $\mathrm{H}(27 \mathrm{~A})-\mathrm{C}(27)-\mathrm{H}(27 \mathrm{~B})$ & 109.0 \\
\hline $\mathrm{C}(20)-\mathrm{C}(21)-\mathrm{H}(21 \mathrm{~A})$ & 111.0 & $\mathrm{C}(28)-\mathrm{C}(27)-\mathrm{C}(26)$ & $104.05(15)$ \\
\hline
\end{tabular}




$\begin{array}{ll}\mathrm{C}(28)-\mathrm{C}(27)-\mathrm{H}(27 \mathrm{~A}) & 110.9 \\ \mathrm{C}(28)-\mathrm{C}(27)-\mathrm{H}(27 \mathrm{~B}) & 110.9 \\ \mathrm{~N}(4)-\mathrm{C}(28)-\mathrm{C}(27) & 103.10(14) \\ \mathrm{N}(4)-\mathrm{C}(28)-\mathrm{H}(28 \mathrm{~A}) & 111.1 \\ \mathrm{~N}(4)-\mathrm{C}(28)-\mathrm{H}(28 \mathrm{~B}) & 111.1 \\ \mathrm{C}(27)-\mathrm{C}(28)-\mathrm{H}(28 \mathrm{~A}) & 111.1 \\ \mathrm{C}(27)-\mathrm{C}(28)-\mathrm{H}(28 \mathrm{~B}) & 111.1 \\ \mathrm{H}(28 \mathrm{~A})-\mathrm{C}(28)-\mathrm{H}(28 \mathrm{~B}) & 109.1 \\ \mathrm{~N}(4)-\mathrm{C}(29)-\mathrm{H}(29 \mathrm{~A}) & 109.5 \\ \mathrm{~N}(4)-\mathrm{C}(29)-\mathrm{H}(29 \mathrm{~B}) & 109.5 \\ \end{array}$

$\begin{array}{ll}\text { N(4)-C(29)-H(29C) } & 109.5 \\ \text { H(29A)-C(29)-H(29B) } & 109.5 \\ \text { H(29A)-C(29)-H(29C) } & 109.5 \\ \text { H(29B)-C(29)-H(29C) } & 109.5 \\ \text { Co(1)-C(4')-H(4'A) } & 102.6 \\ \text { Co(1)-C(4')-H(4'B) } & 116.1 \\ \text { Co(1)-C(4')-H(4'C) } & 109.3 \\ \text { H(4'A)-C(4')-H(4'B) } & 109.5 \\ \text { H(4'A)-C(4')-H(4'C) } & 109.5 \\ \text { H(4'B)-C(4')-H(4'C) } & 109.5\end{array}$




\section{$5.2\left[\mathrm{Mg}(\operatorname{acac})(\mathrm{NMP})_{4}\right]\left[\mathrm{CoMe}{ }_{4}\right][\mathrm{Br}] \cdot 2 \mathrm{NMP}$}

REFERENCE NUMBER: neisc71

CRYSTAL STRUCTURE REPORT

$\mathrm{C}_{64} \mathrm{H}_{118} \mathrm{Br} \mathrm{Co} \mathrm{Mg}_{2} \mathrm{~N}_{10} \mathrm{O}_{14}$

or

$\left[\mathrm{Mg}(\mathrm{acac})(\mathrm{NMP})_{4}\right]_{2}\left[\mathrm{CoMe}_{4}\right][\mathrm{Br}] \cdot 2 \mathrm{NMP}$

Report prepared for:

S. Carpenter, Prof. M. Neidig

October 15, 2018

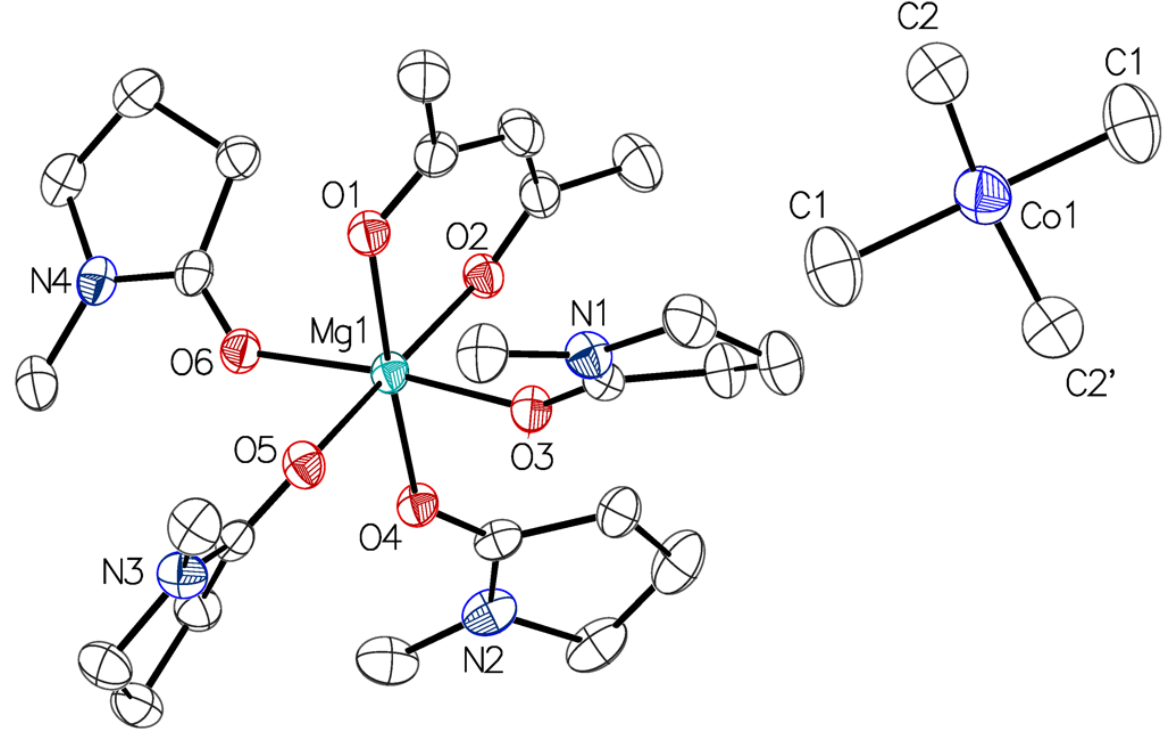

William W. Brennessel

X-ray Crystallographic Facility

Department of Chemistry, University of Rochester

120 Trustee Road

Rochester, NY 14627 


\section{Data collection}

A crystal $\left(0.236 \times 0.198 \times 0.053 \mathrm{~mm}^{3}\right)$ was placed onto a thin glass optical fiber or a nylon loop and mounted on a XtaLab Synergy-S Dualflex diffractometer equipped with a HyPix-6000HE HPC area detector for data collection at 100.00(10) K. A preliminary set of cell constants and an orientation matrix were calculated from a small sampling of reflections. ${ }^{1}$ A short pre-experiment was run, from which an optimal data collection strategy was determined. The full data collection was carried out using a PhotonJet $(\mathrm{Cu})$ X-ray Source with frame times of 0.34 and 1.34 seconds and a detector distance of $31.2 \mathrm{~mm}$. Series of frames were collected in $0.50^{\circ}$ steps in $\omega$ at different $2 \theta$, $\kappa$, and $\phi$ settings. After the intensity data were corrected for absorption, the final cell constants were calculated from the xyz centroids of 13833 strong reflections from the actual data collection after integration. ${ }^{1}$ See Table S9 for additional crystal and refinement information.

\section{Structure solution and refinement}

The structure was solved using ShelXT ${ }^{2}$ and refined using ShelXL. ${ }^{3}$ The space group $P-1$ was determined based on intensity statistics. Most or all non-hydrogen atoms were assigned from the solution. Full-matrix least squares / difference Fourier cycles were performed which located any remaining non-hydrogen atoms. All non-hydrogen atoms were refined with anisotropic displacement parameters. All hydrogen atoms were placed in ideal positions and refined as riding atoms with relative isotropic displacement parameters. The final full matrix least squares refinement converged to $R 1=0.0584\left(F^{2}, I>2 \sigma(I)\right)$ and $w R 2=0.1732\left(F^{2}\right.$, all data).

\section{Structure description}

The structure is the one suggested. The asymmetric unit contains one monocationic Mg complex and one cocrystallized NMP solvent molecule in general positions and one monoanionic tetramethylcobaltate compound and one $\mathrm{Br}$ anion on crystallographic inversion centers. One methyl ligand is modeled as disordered over a crystallographic inversion center (0.50:0.50). One $-\mathrm{CH}_{2}$ - linkage of an NMP ligand is modeled as disordered two positions (0.56:0.44) and the cocrystallized NMP solvent molecule is modeled as disordered over two positions (0.78:0.22). The angle between the C1-Co1-C2 and C1A-Co1-C2'A planes is 13.9(8) degrees.

Structure manipulation and figure generation were performed using Olex $2 .{ }^{4}$ Unless noted otherwise all structural diagrams containing thermal displacement ellipsoids are drawn at the $50 \%$ probability level.

Data collection, structure solution, and structure refinement were conducted at the X-ray Crystallographic Facility, B04 Hutchison Hall, Department of Chemistry, University of Rochester. The instrument was purchased with funding from NSF MRI program grant CHE-1725028. All publications arising from this report MUST either 1) include William W. Brennessel as a coauthor or 2) acknowledge William W. Brennessel and the X-ray Crystallographic Facility of the Department of Chemistry at the University of Rochester. 
1 CrysAlisPro, version 171.39.46; Rigaku Corporation: Oxford, UK, 2018.

2 Sheldrick, G. M. SHELXT, version 2018/2; Acta. Crystallogr. 2015, A71, 3-8.

3 Sheldrick, G. M. SHELXL, version 2018/3; Acta. Crystallogr. 2015, C71, 3-8.

4 Dolomanov, O. V.; Bourhis, L. J.; Gildea, R. J.; Howard, J. A. K.; Puschmann, H. Olex2, version 1.2-ac3; J. Appl. Cryst. 2009, 42, 339-341.

Some equations of interest:

$$
\begin{gathered}
R_{\mathrm{int}}=\Sigma\left|F_{\mathrm{o}}^{2}-<F_{\mathrm{o}}^{2}>\right| / \Sigma\left|F_{\mathrm{o}}{ }^{2}\right| \\
R 1=\Sigma|| F_{\mathrm{o}}|-| F_{\mathrm{c}} \| / \Sigma\left|F_{\mathrm{o}}\right| \\
w R 2=\left[\Sigma\left[w\left(F_{\mathrm{o}}{ }^{2} F_{\mathrm{c}}{ }^{2}\right)^{2}\right] / \Sigma\left[w\left(F_{\mathrm{o}}{ }^{2}\right)^{2}\right]\right]^{1 / 2} \\
\text { where } w=1 /\left[\sigma^{2}\left(F_{\mathrm{o}}{ }^{2}\right)+(a P)^{2}+b P\right] \text { and } \\
P=1 / 3 \max \left(0, F_{\mathrm{o}}{ }^{2}\right)+2 / 3 F_{\mathrm{c}}{ }^{2} \\
\mathrm{GOF}=S=\left[\Sigma\left[w\left(F_{\mathrm{o}}{ }^{2}-F_{\mathrm{c}}{ }^{2}\right)^{2}\right] /(m-n)\right]^{1 / 2}
\end{gathered}
$$

where $m=$ number of reflections and $n=$ number of parameters
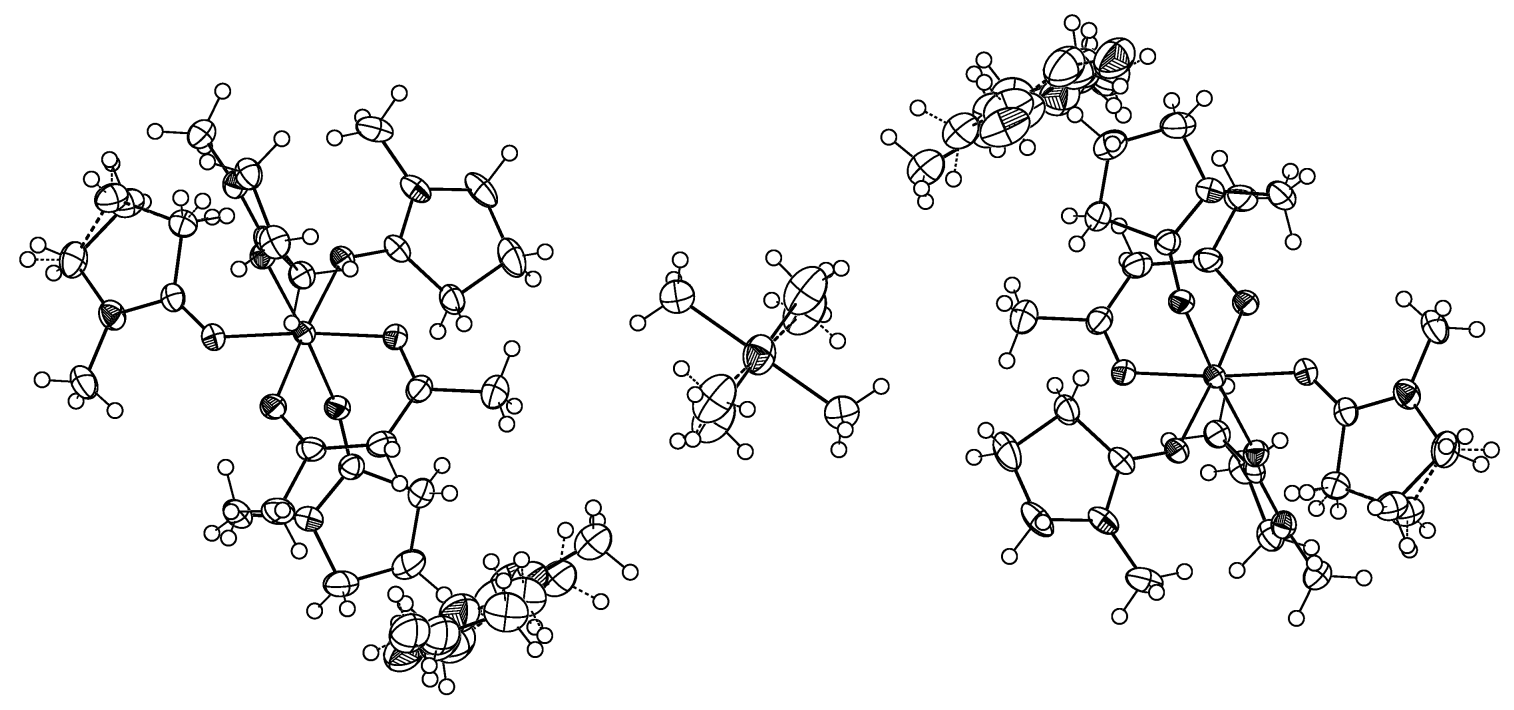

(4) 


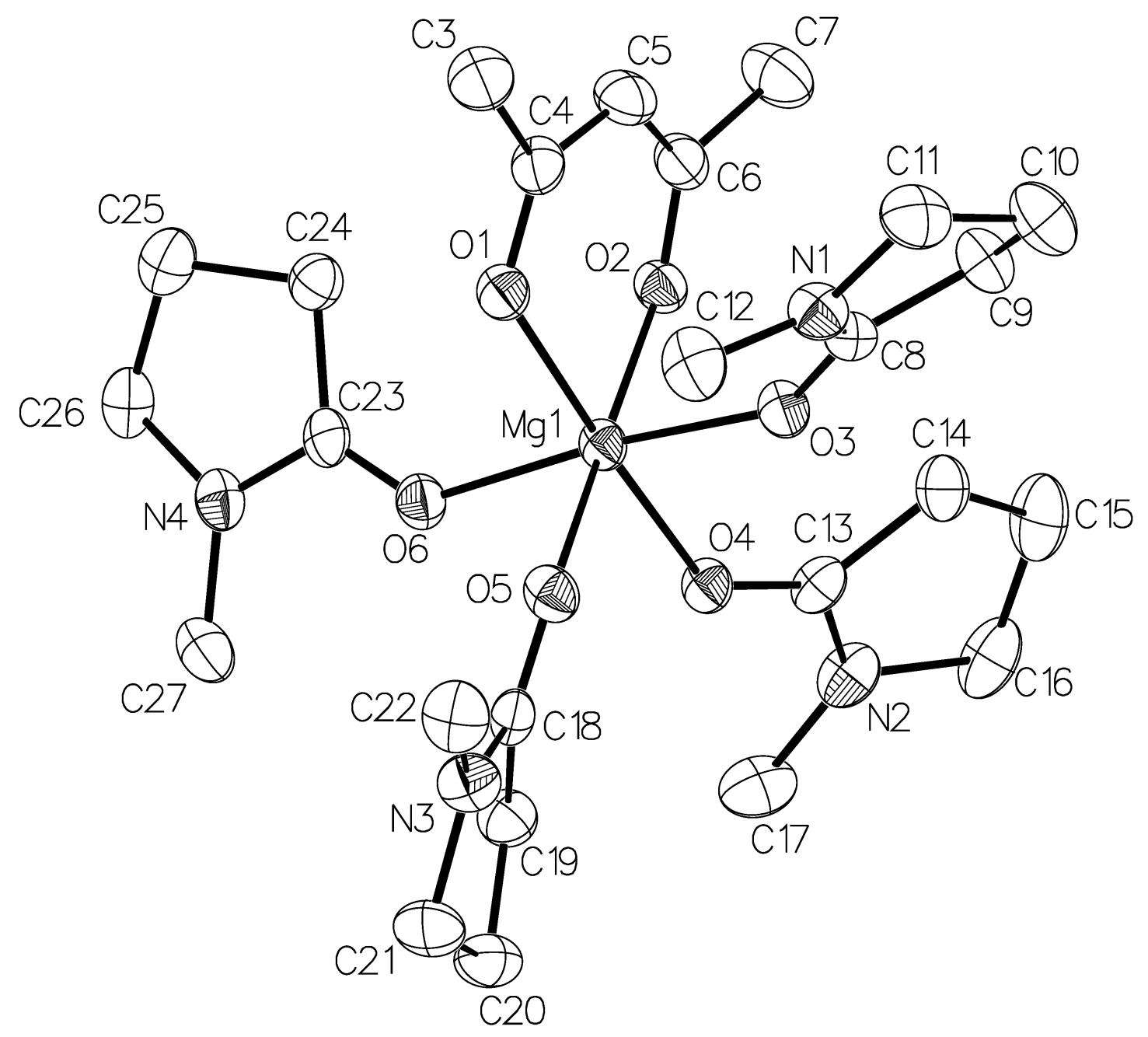



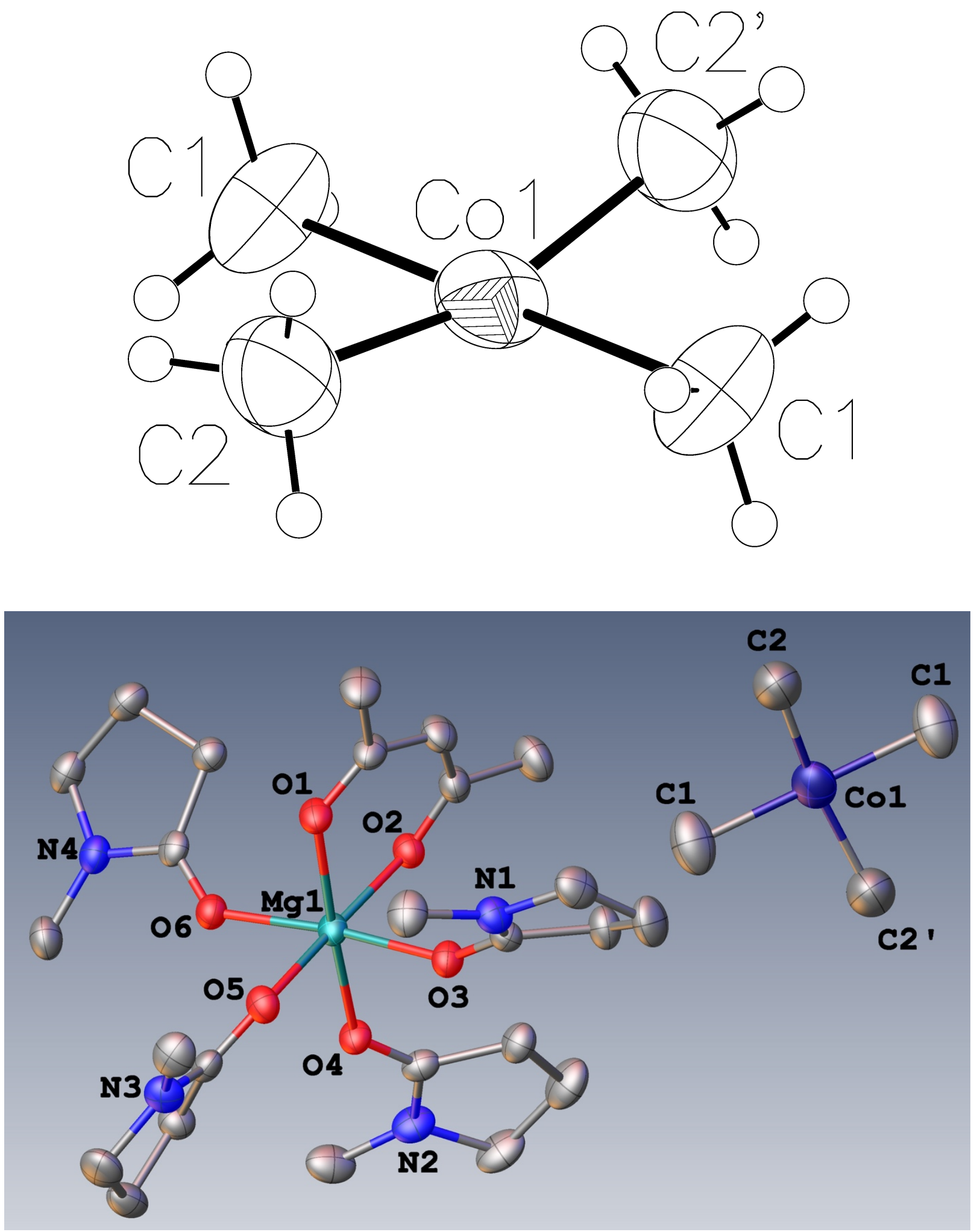
Table S9. Crystal data and structure refinement for neisc71.

\begin{tabular}{|c|c|c|}
\hline Identification code & \multicolumn{2}{|l|}{ neisc 71} \\
\hline Empirical formula & \multicolumn{2}{|c|}{ C64 H118 Br Co Mg2 N10 O14 } \\
\hline Formula weight & \multicolumn{2}{|l|}{1439.14} \\
\hline Temperature & \multicolumn{2}{|l|}{$100.00(10) \mathrm{K}$} \\
\hline Wavelength & \multicolumn{2}{|l|}{$1.54184 \AA$} \\
\hline Crystal system & \multicolumn{2}{|l|}{ triclinic } \\
\hline Space group & \multicolumn{2}{|l|}{$P-1$} \\
\hline \multirow[t]{3}{*}{ Unit cell dimensions } & $a=12.8286(3) \AA$ & $\alpha=69.935(3)^{\circ}$ \\
\hline & $b=13.0310(4) \AA$ & $\beta=68.183(3)^{\circ}$ \\
\hline & $c=13.8035(5) \AA$ & $\gamma=62.522(3)^{\circ}$ \\
\hline Volume & \multicolumn{2}{|l|}{$1858.87(12) \AA^{3}$} \\
\hline$Z$ & \multicolumn{2}{|l|}{1} \\
\hline Density (calculated) & \multicolumn{2}{|l|}{$1.286 \mathrm{Mg} / \mathrm{m}^{3}$} \\
\hline Absorption coefficient & \multicolumn{2}{|l|}{$3.133 \mathrm{~mm}^{-1}$} \\
\hline$F(000)$ & \multicolumn{2}{|l|}{770} \\
\hline Crystal color, morphology & \multicolumn{2}{|l|}{ orange, plate } \\
\hline Crystal size & \multicolumn{2}{|c|}{$0.236 \times 0.198 \times 0.053 \mathrm{~mm}^{3}$} \\
\hline Theta range for data collection & \multicolumn{2}{|l|}{3.526 to $77.807^{\circ}$} \\
\hline Index ranges & \multicolumn{2}{|c|}{$-16 \leq h \leq 15,-15 \leq k \leq 16,-17 \leq l \leq 17$} \\
\hline Reflections collected & \multicolumn{2}{|l|}{30881} \\
\hline Independent reflections & \multicolumn{2}{|c|}{$7792[R($ int $)=0.0518]$} \\
\hline Observed reflections & \multicolumn{2}{|l|}{6602} \\
\hline Completeness to theta $=74.504^{\circ}$ & \multicolumn{2}{|l|}{$99.8 \%$} \\
\hline Absorption correction & \multicolumn{2}{|l|}{ Multi-scan } \\
\hline Max. and min. transmission & \multicolumn{2}{|c|}{1.00000 and 0.66996} \\
\hline Refinement method & \multicolumn{2}{|c|}{ Full-matrix least-squares on $F^{2}$} \\
\hline Data / restraints / parameters & \multicolumn{2}{|l|}{7792 / 24 / 446} \\
\hline Goodness-of-fit on $F^{2}$ & \multicolumn{2}{|l|}{1.088} \\
\hline Final $R$ indices $[I>2 \operatorname{sigma}(I)]$ & \multicolumn{2}{|c|}{$R 1=0.0584, w R 2=0.1668$} \\
\hline$R$ indices (all data) & \multicolumn{2}{|c|}{$R 1=0.0656, w R 2=0.1732$} \\
\hline Largest diff. peak and hole & \multicolumn{2}{|c|}{0.677 and -0.691 e. $\AA^{-3}$} \\
\hline
\end{tabular}


Table S10. Atomic coordinates $\left(\times 10^{4}\right)$ and equivalent isotropic displacement parameters $\left(\AA^{2} \times 10^{3}\right)$ for neisc71. $U_{e q}$ is defined as one third of the trace of the orthogonalized $U_{i j}$ tensor.

\begin{tabular}{|c|c|c|c|c|}
\hline & $\mathrm{x}$ & $\mathrm{y}$ & $\mathrm{z}$ & $\mathrm{U}_{\mathrm{eq}}$ \\
\hline $\mathrm{Br} 1$ & 5000 & 0 & 10000 & $51(1)$ \\
\hline Co1 & 0 & 5000 & 5000 & $52(1)$ \\
\hline $\mathrm{Mg} 1$ & 7994(1) & $2876(1)$ & $1514(1)$ & $24(1)$ \\
\hline $\mathrm{O} 1$ & $8785(2)$ & $1295(1)$ & 2482(1) & $30(1)$ \\
\hline $\mathrm{O} 2$ & $6388(1)$ & $2643(1)$ & 2292(1) & $27(1)$ \\
\hline $\mathrm{O} 3$ & $7670(1)$ & $3751(1)$ & 2666(1) & $28(1)$ \\
\hline $\mathrm{O} 4$ & $7075(1)$ & $4445(1)$ & $600(1)$ & $27(1)$ \\
\hline O5 & $9627(1)$ & $3088(2)$ & $855(1)$ & $29(1)$ \\
\hline O6 & $8456(1)$ & $2087(1)$ & $259(1)$ & $28(1)$ \\
\hline $\mathrm{O} 7$ & 5124(4) & 2676(3) & 6507(3) & $68(1)$ \\
\hline $\mathrm{C} 28$ & $5434(7)$ & $1576(5)$ & $6739(5)$ & $68(1)$ \\
\hline C29 & $6623(6)$ & $729(8)$ & 6985(8) & $76(2)$ \\
\hline C30 & $6606(6)$ & $-465(6)$ & 7064(5) & $69(1)$ \\
\hline $\mathrm{C} 31$ & $5344(5)$ & $-266(4)$ & $7231(5)$ & $63(1)$ \\
\hline N5 & $4828(4)$ & $969(4)$ & 6810(3) & $58(1)$ \\
\hline C32 & 3571(4) & $1579(5)$ & $6705(4)$ & $58(1)$ \\
\hline O7' & $7295(12)$ & $1110(14)$ & $6811(10)$ & $76(2)$ \\
\hline $\mathrm{C} 28^{\prime}$ & 6371(19) & $900(20)$ & $7100(30)$ & $76(2)$ \\
\hline C29' & $5250(20)$ & $1896(17)$ & $6830(20)$ & $68(1)$ \\
\hline C30' & $4321(15)$ & $1438(16)$ & $6952(15)$ & $58(1)$ \\
\hline C31' & 4979(16) & $190(15)$ & $7100(20)$ & $63(1)$ \\
\hline N5' & $6179(13)$ & $150(13)$ & $6839(13)$ & $69(1)$ \\
\hline C32' & $7125(17)$ & $-1055(16)$ & $6883(16)$ & $69(1)$ \\
\hline N1 & $8554(2)$ & $3205(2)$ & 4025(2) & $31(1)$ \\
\hline $\mathrm{N} 2$ & $5546(2)$ & $6060(2)$ & $5(2)$ & $34(1)$ \\
\hline N3 & $11351(2)$ & $3332(2)$ & $-244(2)$ & $33(1)$ \\
\hline N4 & $8691(2)$ & $908(2)$ & $-764(2)$ & $30(1)$ \\
\hline $\mathrm{C} 1$ & $-1264(3)$ & $5098(4)$ & 6399(3) & $65(1)$ \\
\hline $\mathrm{C} 2$ & $1020(20)$ & $3451(8)$ & $5716(16)$ & $77(4)$ \\
\hline $\mathrm{C} 2^{\prime}$ & $940(20)$ & $3296(8)$ & $5469(16)$ & $77(4)$ \\
\hline C3 & $9050(3)$ & $-91(3)$ & 4107(2) & $43(1)$ \\
\hline
\end{tabular}




\begin{tabular}{|c|c|c|c|c|}
\hline $\mathrm{C} 4$ & $8255(2)$ & $885(2)$ & $3398(2)$ & $31(1)$ \\
\hline $\mathrm{C} 5$ & 6990(2) & $1253(2)$ & $3816(2)$ & $36(1)$ \\
\hline C6 & $6135(2)$ & $2106(2)$ & $3247(2)$ & $32(1)$ \\
\hline $\mathrm{C} 7$ & $4806(3)$ & $2438(3)$ & $3825(2)$ & $46(1)$ \\
\hline $\mathrm{C} 8$ & $7622(2)$ & $3723(2)$ & $3580(2)$ & $26(1)$ \\
\hline C9 & $6482(2)$ & $4285(3)$ & $4388(2)$ & $36(1)$ \\
\hline $\mathrm{C} 10$ & 6949(3) & $4275(3)$ & $5261(2)$ & $48(1)$ \\
\hline C11 & $8208(3)$ & $3288(3)$ & $5138(2)$ & $41(1)$ \\
\hline $\mathrm{C} 12$ & $9796(2)$ & $2550(3)$ & $3500(2)$ & $41(1)$ \\
\hline $\mathrm{C} 13$ & $6070(2)$ & $5275(2)$ & $774(2)$ & $27(1)$ \\
\hline $\mathrm{C} 14$ & $5273(2)$ & $5585(2)$ & $1840(2)$ & $32(1)$ \\
\hline $\mathrm{C} 15$ & $4078(3)$ & 6539(3) & $1605(3)$ & $49(1)$ \\
\hline C16 & $4389(3)$ & 6992(2) & $400(3)$ & $44(1)$ \\
\hline $\mathrm{C} 17$ & $6069(3)$ & $6074(3)$ & $-1115(2)$ & $42(1)$ \\
\hline $\mathrm{C} 18$ & $10208(2)$ & $3446(2)$ & $-35(2)$ & $25(1)$ \\
\hline C19 & $9779(2)$ & $4076(2)$ & $-1038(2)$ & $33(1)$ \\
\hline $\mathrm{C} 20$ & $10698(4)$ & $4582(5)$ & $-1802(4)$ & $37(1)$ \\
\hline $\mathrm{C} 20^{\prime}$ & 10961(5) & 3991(7) & $-1921(5)$ & $37(1)$ \\
\hline $\mathrm{C} 21$ & $11848(2)$ & $3829(3)$ & $-1351(2)$ & $42(1)$ \\
\hline $\mathrm{C} 22$ & $12077(2)$ & 2733(3) & $526(2)$ & $40(1)$ \\
\hline $\mathrm{C} 23$ & $8360(2)$ & $1222(2)$ & $162(2)$ & $26(1)$ \\
\hline $\mathrm{C} 24$ & $7819(2)$ & $397(2)$ & $1036(2)$ & $30(1)$ \\
\hline $\mathrm{C} 25$ & $8176(3)$ & $-622(2)$ & $512(2)$ & $38(1)$ \\
\hline $\mathrm{C} 26$ & $8413(2)$ & $-93(2)$ & $-679(2)$ & $35(1)$ \\
\hline $\mathrm{C} 27$ & $9123(2)$ & $1586(3)$ & $-1791(2)$ & $38(1)$ \\
\hline
\end{tabular}


Table S11. Bond lengths $[\AA]$ and angles $\left[{ }^{\circ}\right]$ for neisc 71 .

\begin{tabular}{|c|c|c|c|}
\hline $\mathrm{Co}(1)-\mathrm{C}(1)$ & $2.006(4)$ & $\mathrm{O}\left(7^{\prime}\right)-\mathrm{C}\left(28^{\prime}\right)$ & $1.229(18)$ \\
\hline $\mathrm{Co}(1)-\mathrm{C}(1) \# 1$ & $2.006(4)$ & $\mathrm{C}\left(28^{\prime}\right)-\mathrm{C}\left(29^{\prime}\right)$ & $1.497(17)$ \\
\hline $\mathrm{Co}(1)-\mathrm{C}(2) \# 1$ & $1.982(8)$ & $\mathrm{C}\left(28^{\prime}\right)-\mathrm{N}\left(5^{\prime}\right)$ & $1.299(12)$ \\
\hline $\mathrm{Co}(1)-\mathrm{C}(2)$ & $1.982(8)$ & $\mathrm{C}\left(29^{\prime}\right)-\mathrm{H}(29 \mathrm{C})$ & 0.9700 \\
\hline $\mathrm{Co}(1)-\mathrm{C}\left(2^{\prime}\right) \# 1$ & $1.981(7)$ & $\mathrm{C}\left(29^{\prime}\right)-\mathrm{H}(29 \mathrm{D})$ & 0.9700 \\
\hline $\mathrm{Co}(1)-\mathrm{C}\left(2^{\prime}\right)$ & $1.981(7)$ & $\mathrm{C}\left(29^{\prime}\right)-\mathrm{C}\left(30^{\prime}\right)$ & $1.496(19)$ \\
\hline $\mathrm{Mg}(1)-\mathrm{O}(1)$ & $2.0610(17)$ & $\mathrm{C}\left(30^{\prime}\right)-\mathrm{H}(30 \mathrm{C})$ & 0.9700 \\
\hline $\mathrm{Mg}(1)-\mathrm{O}(2)$ & $2.0499(18)$ & $\mathrm{C}\left(30^{\prime}\right)-\mathrm{H}(30 \mathrm{D})$ & 0.9700 \\
\hline $\mathrm{Mg}(1)-\mathrm{O}(3)$ & $2.0769(18)$ & $\mathrm{C}\left(30^{\prime}\right)-\mathrm{C}\left(31^{\prime}\right)$ & $1.426(17)$ \\
\hline $\mathrm{Mg}(1)-\mathrm{O}(4)$ & $2.0674(16)$ & $\mathrm{C}\left(31^{\prime}\right)-\mathrm{H}(31 \mathrm{C})$ & 0.9700 \\
\hline $\mathrm{Mg}(1)-\mathrm{O}(5)$ & $2.0575(18)$ & C(31')-H(31D) & 0.9700 \\
\hline $\mathrm{Mg}(1)-\mathrm{O}(6)$ & $2.0762(18)$ & $\mathrm{C}\left(31^{\prime}\right)-\mathrm{N}\left(5^{\prime}\right)$ & $1.424(16)$ \\
\hline $\mathrm{O}(1)-\mathrm{C}(4)$ & $1.257(3)$ & $\mathrm{N}\left(5^{\prime}\right)-\mathrm{C}\left(32^{\prime}\right)$ & $1.474(16)$ \\
\hline $\mathrm{O}(2)-\mathrm{C}(6)$ & $1.261(3)$ & $\mathrm{C}\left(32^{\prime}\right)-\mathrm{H}(32 \mathrm{D})$ & 0.9600 \\
\hline $\mathrm{O}(3)-\mathrm{C}(8)$ & $1.229(3)$ & $\mathrm{C}\left(32^{\prime}\right)-\mathrm{H}(32 \mathrm{E})$ & 0.9600 \\
\hline $\mathrm{O}(4)-\mathrm{C}(13)$ & $1.246(3)$ & $\mathrm{C}\left(32^{\prime}\right)-\mathrm{H}(32 \mathrm{~F})$ & 0.9600 \\
\hline $\mathrm{O}(5)-\mathrm{C}(18)$ & $1.237(3)$ & $\mathrm{N}(1)-\mathrm{C}(8)$ & $1.334(3)$ \\
\hline $\mathrm{O}(6)-\mathrm{C}(23)$ & $1.244(3)$ & $\mathrm{N}(1)-\mathrm{C}(11)$ & $1.460(3)$ \\
\hline $\mathrm{O}(7)-\mathrm{C}(28)$ & $1.253(6)$ & $\mathrm{N}(1)-\mathrm{C}(12)$ & $1.451(3)$ \\
\hline$C(28)-C(29)$ & $1.494(9)$ & $\mathrm{N}(2)-\mathrm{C}(13)$ & $1.332(3)$ \\
\hline $\mathrm{C}(28)-\mathrm{N}(5)$ & $1.303(8)$ & $\mathrm{N}(2)-\mathrm{C}(16)$ & $1.471(3)$ \\
\hline $\mathrm{C}(29)-\mathrm{H}(29 \mathrm{~A})$ & 0.9700 & $\mathrm{~N}(2)-\mathrm{C}(17)$ & $1.436(4)$ \\
\hline $\mathrm{C}(29)-\mathrm{H}(29 \mathrm{~B})$ & 0.9700 & $\mathrm{~N}(3)-\mathrm{C}(18)$ & $1.329(3)$ \\
\hline C(29)-C(30) & $1.531(10)$ & $\mathrm{N}(3)-\mathrm{C}(21)$ & $1.463(4)$ \\
\hline $\mathrm{C}(30)-\mathrm{H}(30 \mathrm{~A})$ & 0.9700 & $\mathrm{~N}(3)-\mathrm{C}(22)$ & $1.450(3)$ \\
\hline $\mathrm{C}(30)-\mathrm{H}(30 \mathrm{~B})$ & 0.9700 & $\mathrm{~N}(4)-\mathrm{C}(23)$ & $1.332(3)$ \\
\hline $\mathrm{C}(30)-\mathrm{C}(31)$ & $1.456(8)$ & $\mathrm{N}(4)-\mathrm{C}(26)$ & $1.461(3)$ \\
\hline $\mathrm{C}(31)-\mathrm{H}(31 \mathrm{~A})$ & 0.9700 & $\mathrm{~N}(4)-\mathrm{C}(27)$ & $1.452(3)$ \\
\hline $\mathrm{C}(31)-\mathrm{H}(31 \mathrm{~B})$ & 0.9700 & $\mathrm{C}(1)-\mathrm{H}(1 \mathrm{~A})$ & 0.9600 \\
\hline C(31)-N(5) & $1.423(6)$ & C(1)-H(1B) & 0.9600 \\
\hline N(5)-C(32) & $1.471(7)$ & C(1)-H(1C) & 0.9600 \\
\hline $\mathrm{C}(32)-\mathrm{H}(32 \mathrm{~A})$ & 0.9600 & $\mathrm{C}(2)-\mathrm{H}(2 \mathrm{~A})$ & 0.9600 \\
\hline $\mathrm{C}(32)-\mathrm{H}(32 \mathrm{~B})$ & 0.9600 & $\mathrm{C}(2)-\mathrm{H}(2 \mathrm{~B})$ & 0.9600 \\
\hline $\mathrm{C}(32)-\mathrm{H}(32 \mathrm{C})$ & 0.9600 & $\mathrm{C}(2)-\mathrm{H}(2 \mathrm{C})$ & 0.9600 \\
\hline
\end{tabular}




\begin{tabular}{|c|c|c|c|}
\hline $\mathrm{C}\left(2^{\prime}\right)-\mathrm{H}\left(2^{\prime} \mathrm{A}\right)$ & 0.9600 & $\mathrm{C}(17)-\mathrm{H}(17 \mathrm{~A})$ & 0.9600 \\
\hline $\mathrm{C}\left(2^{\prime}\right)-\mathrm{H}\left(2^{\prime} \mathrm{B}\right)$ & 0.9600 & $\mathrm{C}(17)-\mathrm{H}(17 \mathrm{~B})$ & 0.9600 \\
\hline $\mathrm{C}\left(2^{\prime}\right)-\mathrm{H}\left(2^{\prime} \mathrm{C}\right)$ & 0.9600 & $\mathrm{C}(17)-\mathrm{H}(17 \mathrm{C})$ & 0.9600 \\
\hline $\mathrm{C}(3)-\mathrm{H}(3 \mathrm{~A})$ & 0.9600 & $\mathrm{C}(18)-\mathrm{C}(19)$ & $1.504(3)$ \\
\hline $\mathrm{C}(3)-\mathrm{H}(3 \mathrm{~B})$ & 0.9600 & C(19)-H(19A) & 0.9700 \\
\hline $\mathrm{C}(3)-\mathrm{H}(3 \mathrm{C})$ & 0.9600 & C(19)-H(19B) & 0.9700 \\
\hline$C(3)-C(4)$ & $1.518(3)$ & C(19)-H(19C) & 0.9700 \\
\hline$C(4)-C(5)$ & $1.404(4)$ & C(19)-H(19D) & 0.9700 \\
\hline $\mathrm{C}(5)-\mathrm{H}(5 \mathrm{~A})$ & 0.9700 & $\mathrm{C}(19)-\mathrm{C}(20)$ & $1.501(5)$ \\
\hline $\mathrm{C}(5)-\mathrm{H}(5 \mathrm{~B})$ & 0.9700 & $\mathrm{C}(19)-\mathrm{C}\left(20^{\prime}\right)$ & $1.537(6)$ \\
\hline$C(5)-C(6)$ & $1.405(3)$ & $\mathrm{C}(20)-\mathrm{H}(20 \mathrm{~A})$ & 0.9700 \\
\hline$C(6)-C(7)$ & $1.508(4)$ & $\mathrm{C}(20)-\mathrm{H}(20 \mathrm{~B})$ & 0.9700 \\
\hline $\mathrm{C}(7)-\mathrm{H}(7 \mathrm{~A})$ & 0.9600 & $\mathrm{C}(20)-\mathrm{C}(21)$ & $1.567(5)$ \\
\hline $\mathrm{C}(7)-\mathrm{H}(7 \mathrm{~B})$ & 0.9600 & $\mathrm{C}\left(20^{\prime}\right)-\mathrm{H}(20 \mathrm{C})$ & 0.9700 \\
\hline $\mathrm{C}(7)-\mathrm{H}(7 \mathrm{C})$ & 0.9600 & $\mathrm{C}\left(20^{\prime}\right)-\mathrm{H}(20 \mathrm{D})$ & 0.9700 \\
\hline $\mathrm{C}(8)-\mathrm{C}(9)$ & $1.509(3)$ & $\mathrm{C}\left(20^{\prime}\right)-\mathrm{C}(21)$ & $1.514(6)$ \\
\hline $\mathrm{C}(9)-\mathrm{H}(9 \mathrm{~A})$ & 0.9700 & $\mathrm{C}(21)-\mathrm{H}(21 \mathrm{~A})$ & 0.9700 \\
\hline $\mathrm{C}(9)-\mathrm{H}(9 \mathrm{~B})$ & 0.9700 & $\mathrm{C}(21)-\mathrm{H}(21 \mathrm{~B})$ & 0.9700 \\
\hline $\mathrm{C}(9)-\mathrm{C}(10)$ & $1.526(4)$ & $\mathrm{C}(21)-\mathrm{H}(21 \mathrm{C})$ & 0.9700 \\
\hline $\mathrm{C}(10)-\mathrm{H}(10 \mathrm{~A})$ & 0.9700 & $\mathrm{C}(21)-\mathrm{H}(21 \mathrm{D})$ & 0.9700 \\
\hline $\mathrm{C}(10)-\mathrm{H}(10 \mathrm{~B})$ & 0.9700 & $\mathrm{C}(22)-\mathrm{H}(22 \mathrm{~A})$ & 0.9600 \\
\hline$C(10)-C(11)$ & $1.523(4)$ & $\mathrm{C}(22)-\mathrm{H}(22 \mathrm{~B})$ & 0.9600 \\
\hline $\mathrm{C}(11)-\mathrm{H}(11 \mathrm{~A})$ & 0.9700 & $\mathrm{C}(22)-\mathrm{H}(22 \mathrm{C})$ & 0.9600 \\
\hline $\mathrm{C}(11)-\mathrm{H}(11 \mathrm{~B})$ & 0.9700 & $C(23)-C(24)$ & $1.507(3)$ \\
\hline $\mathrm{C}(12)-\mathrm{H}(12 \mathrm{~A})$ & 0.9600 & $\mathrm{C}(24)-\mathrm{H}(24 \mathrm{~A})$ & 0.9700 \\
\hline $\mathrm{C}(12)-\mathrm{H}(12 \mathrm{~B})$ & 0.9600 & $\mathrm{C}(24)-\mathrm{H}(24 \mathrm{~B})$ & 0.9700 \\
\hline $\mathrm{C}(12)-\mathrm{H}(12 \mathrm{C})$ & 0.9600 & $\mathrm{C}(24)-\mathrm{C}(25)$ & $1.532(4)$ \\
\hline$C(13)-C(14)$ & $1.511(3)$ & $\mathrm{C}(25)-\mathrm{H}(25 \mathrm{~A})$ & 0.9700 \\
\hline $\mathrm{C}(14)-\mathrm{H}(14 \mathrm{~A})$ & 0.9700 & $\mathrm{C}(25)-\mathrm{H}(25 \mathrm{~B})$ & 0.9700 \\
\hline $\mathrm{C}(14)-\mathrm{H}(14 \mathrm{~B})$ & 0.9700 & $\mathrm{C}(25)-\mathrm{C}(26)$ & $1.522(4)$ \\
\hline$C(14)-C(15)$ & $1.528(3)$ & $\mathrm{C}(26)-\mathrm{H}(26 \mathrm{~A})$ & 0.9700 \\
\hline $\mathrm{C}(15)-\mathrm{H}(15 \mathrm{~A})$ & 0.9700 & $\mathrm{C}(26)-\mathrm{H}(26 \mathrm{~B})$ & 0.9700 \\
\hline $\mathrm{C}(15)-\mathrm{H}(15 \mathrm{~B})$ & 0.9700 & $\mathrm{C}(27)-\mathrm{H}(27 \mathrm{~A})$ & 0.9600 \\
\hline$C(15)-C(16)$ & $1.525(5)$ & $\mathrm{C}(27)-\mathrm{H}(27 \mathrm{~B})$ & 0.9600 \\
\hline $\mathrm{C}(16)-\mathrm{H}(16 \mathrm{~A})$ & 0.9700 & $\mathrm{C}(27)-\mathrm{H}(27 \mathrm{C})$ & 0.9600 \\
\hline $\mathrm{C}(16)-\mathrm{H}(16 \mathrm{~B})$ & 0.9700 & & \\
\hline
\end{tabular}




\begin{tabular}{|c|c|}
\hline $\mathrm{C}(1) \# 1-\mathrm{Co}(1)-\mathrm{C}(1)$ & 180.0 \\
\hline $\mathrm{C}(2) \# 1-\mathrm{Co}(1)-\mathrm{C}(1) \# 1$ & $87.7(7)$ \\
\hline $\mathrm{C}(2) \# 1-\mathrm{Co}(1)-\mathrm{C}(1)$ & $92.3(7)$ \\
\hline $\mathrm{C}(2)-\mathrm{Co}(1)-\mathrm{C}(1)$ & $87.7(7)$ \\
\hline $\mathrm{C}(2)-\mathrm{Co}(1)-\mathrm{C}(1) \# 1$ & $92.3(7)$ \\
\hline $\mathrm{C}(2) \# 1-\mathrm{Co}(1)-\mathrm{C}(2)$ & $180.0(14)$ \\
\hline $\mathrm{C}\left(2^{\prime}\right) \# 1-\mathrm{Co}(1)-\mathrm{C}(1)$ & $86.8(7)$ \\
\hline $\mathrm{C}\left(2^{\prime}\right)-\mathrm{Co}(1)-\mathrm{C}(1)$ & $93.2(7)$ \\
\hline $\mathrm{C}\left(2^{\prime}\right)-\mathrm{Co}(1)-\mathrm{C}\left(2^{\prime}\right) \# 1$ & 180.0 \\
\hline $\mathrm{O}(1)-\mathrm{Mg}(1)-\mathrm{O}(3)$ & $89.05(7)$ \\
\hline $\mathrm{O}(1)-\mathrm{Mg}(1)-\mathrm{O}(4)$ & $175.57(8)$ \\
\hline $\mathrm{O}(1)-\mathrm{Mg}(1)-\mathrm{O}(6)$ & $93.26(7)$ \\
\hline $\mathrm{O}(2)-\mathrm{Mg}(1)-\mathrm{O}(1)$ & $86.34(7)$ \\
\hline $\mathrm{O}(2)-\mathrm{Mg}(1)-\mathrm{O}(3)$ & $89.15(7)$ \\
\hline $\mathrm{O}(2)-\mathrm{Mg}(1)-\mathrm{O}(4)$ & $89.23(7)$ \\
\hline $\mathrm{O}(2)-\mathrm{Mg}(1)-\mathrm{O}(5)$ & $175.28(8)$ \\
\hline $\mathrm{O}(2)-\mathrm{Mg}(1)-\mathrm{O}(6)$ & $96.86(7)$ \\
\hline $\mathrm{O}(4)-\mathrm{Mg}(1)-\mathrm{O}(3)$ & $91.03(7)$ \\
\hline $\mathrm{O}(4)-\mathrm{Mg}(1)-\mathrm{O}(6)$ & $87.12(7)$ \\
\hline $\mathrm{O}(5)-\mathrm{Mg}(1)-\mathrm{O}(1)$ & $90.74(7)$ \\
\hline $\mathrm{O}(5)-\mathrm{Mg}(1)-\mathrm{O}(3)$ & $87.08(7)$ \\
\hline $\mathrm{O}(5)-\mathrm{Mg}(1)-\mathrm{O}(4)$ & $93.69(7)$ \\
\hline $\mathrm{O}(5)-\mathrm{Mg}(1)-\mathrm{O}(6)$ & $87.00(7)$ \\
\hline $\mathrm{O}(6)-\mathrm{Mg}(1)-\mathrm{O}(3)$ & $173.68(8)$ \\
\hline $\mathrm{C}(4)-\mathrm{O}(1)-\mathrm{Mg}(1)$ & $125.31(15)$ \\
\hline $\mathrm{C}(6)-\mathrm{O}(2)-\mathrm{Mg}(1)$ & $124.74(16)$ \\
\hline $\mathrm{C}(8)-\mathrm{O}(3)-\mathrm{Mg}(1)$ & $149.81(16)$ \\
\hline $\mathrm{C}(13)-\mathrm{O}(4)-\mathrm{Mg}(1)$ & $135.10(16)$ \\
\hline $\mathrm{C}(18)-\mathrm{O}(5)-\mathrm{Mg}(1)$ & $137.76(15)$ \\
\hline $\mathrm{C}(23)-\mathrm{O}(6)-\mathrm{Mg}(1)$ & $135.33(16)$ \\
\hline $\mathrm{O}(7)-\mathrm{C}(28)-\mathrm{C}(29)$ & $123.4(7)$ \\
\hline $\mathrm{O}(7)-\mathrm{C}(28)-\mathrm{N}(5)$ & $128.3(7)$ \\
\hline $\mathrm{N}(5)-\mathrm{C}(28)-\mathrm{C}(29)$ & $108.3(5)$ \\
\hline $\mathrm{C}(28)-\mathrm{C}(29)-\mathrm{H}(29 \mathrm{~A})$ & 111.4 \\
\hline C(28)-C(29)-H(29B) & 111.4 \\
\hline $\mathrm{C}(28)-\mathrm{C}(29)-\mathrm{C}(30)$ & $102.0(5)$ \\
\hline
\end{tabular}

\begin{tabular}{|c|c|}
\hline H(29A)-C(29)-H(29B) & 109.2 \\
\hline $\mathrm{C}(30)-\mathrm{C}(29)-\mathrm{H}(29 \mathrm{~A})$ & 111.4 \\
\hline $\mathrm{C}(30)-\mathrm{C}(29)-\mathrm{H}(29 \mathrm{~B})$ & 111.4 \\
\hline $\mathrm{C}(29)-\mathrm{C}(30)-\mathrm{H}(30 \mathrm{~A})$ & 110.4 \\
\hline $\mathrm{C}(29)-\mathrm{C}(30)-\mathrm{H}(30 \mathrm{~B})$ & 110.4 \\
\hline $\mathrm{H}(30 \mathrm{~A})-\mathrm{C}(30)-\mathrm{H}(30 \mathrm{~B})$ & 108.6 \\
\hline$C(31)-C(30)-C(29)$ & $106.6(5)$ \\
\hline $\mathrm{C}(31)-\mathrm{C}(30)-\mathrm{H}(30 \mathrm{~A})$ & 110.4 \\
\hline $\mathrm{C}(31)-\mathrm{C}(30)-\mathrm{H}(30 \mathrm{~B})$ & 110.4 \\
\hline $\mathrm{C}(30)-\mathrm{C}(31)-\mathrm{H}(31 \mathrm{~A})$ & 111.3 \\
\hline $\mathrm{C}(30)-\mathrm{C}(31)-\mathrm{H}(31 \mathrm{~B})$ & 111.3 \\
\hline $\mathrm{H}(31 \mathrm{~A})-\mathrm{C}(31)-\mathrm{H}(31 \mathrm{~B})$ & 109.2 \\
\hline $\mathrm{N}(5)-\mathrm{C}(31)-\mathrm{C}(30)$ & $102.3(4)$ \\
\hline $\mathrm{N}(5)-\mathrm{C}(31)-\mathrm{H}(31 \mathrm{~A})$ & 111.3 \\
\hline $\mathrm{N}(5)-\mathrm{C}(31)-\mathrm{H}(31 \mathrm{~B})$ & 111.3 \\
\hline$C(28)-N(5)-C(31)$ & $115.4(5)$ \\
\hline $\mathrm{C}(28)-\mathrm{N}(5)-\mathrm{C}(32)$ & $120.2(5)$ \\
\hline$C(31)-N(5)-C(32)$ & $122.8(4)$ \\
\hline $\mathrm{N}(5)-\mathrm{C}(32)-\mathrm{H}(32 \mathrm{~A})$ & 109.5 \\
\hline $\mathrm{N}(5)-\mathrm{C}(32)-\mathrm{H}(32 \mathrm{~B})$ & 109.5 \\
\hline $\mathrm{N}(5)-\mathrm{C}(32)-\mathrm{H}(32 \mathrm{C})$ & 109.5 \\
\hline $\mathrm{H}(32 \mathrm{~A})-\mathrm{C}(32)-\mathrm{H}(32 \mathrm{~B})$ & 109.5 \\
\hline $\mathrm{H}(32 \mathrm{~A})-\mathrm{C}(32)-\mathrm{H}(32 \mathrm{C})$ & 109.5 \\
\hline $\mathrm{H}(32 \mathrm{~B})-\mathrm{C}(32)-\mathrm{H}(32 \mathrm{C})$ & 109.5 \\
\hline $\mathrm{O}\left(7^{\prime}\right)-\mathrm{C}\left(28^{\prime}\right)-\mathrm{C}\left(29^{\prime}\right)$ & $117(2)$ \\
\hline $\mathrm{O}\left(7^{\prime}\right)-\mathrm{C}\left(28^{\prime}\right)-\mathrm{N}\left(5^{\prime}\right)$ & $129(2)$ \\
\hline $\mathrm{N}\left(5^{\prime}\right)-\mathrm{C}\left(28^{\prime}\right)-\mathrm{C}\left(2^{\prime} 9^{\prime}\right)$ & $92.4(14)$ \\
\hline $\mathrm{C}\left(28^{\prime}\right)-\mathrm{C}\left(29^{\prime}\right)-\mathrm{H}(29 \mathrm{C})$ & 109.5 \\
\hline $\mathrm{C}\left(28^{\prime}\right)-\mathrm{C}\left(29^{\prime}\right)-\mathrm{H}(29 \mathrm{D})$ & 109.5 \\
\hline $\mathrm{H}(29 \mathrm{C})-\mathrm{C}\left(29^{\prime}\right)-\mathrm{H}(29 \mathrm{D})$ & 108.1 \\
\hline $\mathrm{C}\left(30^{\prime}\right)-\mathrm{C}\left(29^{\prime}\right)-\mathrm{C}\left(28^{\prime}\right)$ & $110.7(15)$ \\
\hline $\mathrm{C}\left(30^{\prime}\right)-\mathrm{C}\left(29^{\prime}\right)-\mathrm{H}(29 \mathrm{C})$ & 109.5 \\
\hline $\mathrm{C}\left(30^{\prime}\right)-\mathrm{C}\left(29^{\prime}\right)-\mathrm{H}(29 \mathrm{D})$ & 109.5 \\
\hline $\mathrm{C}\left(29^{\prime}\right)-\mathrm{C}\left(30^{\prime}\right)-\mathrm{H}(30 \mathrm{C})$ & 111.1 \\
\hline $\mathrm{C}\left(29^{\prime}\right)-\mathrm{C}\left(30^{\prime}\right)-\mathrm{H}(30 \mathrm{D})$ & 111.1 \\
\hline $\mathrm{H}(30 \mathrm{C})-\mathrm{C}\left(30^{\prime}\right)-\mathrm{H}(30 \mathrm{D})$ & 109.1 \\
\hline
\end{tabular}




\begin{tabular}{|c|c|c|c|}
\hline $\mathrm{C}\left(31^{\prime}\right)-\mathrm{C}\left(30^{\prime}\right)-\mathrm{C}\left(29^{\prime}\right)$ & $103.1(13)$ & $\mathrm{Co}(1)-\mathrm{C}(2)-\mathrm{H}(2 \mathrm{~A})$ & 109.5 \\
\hline $\mathrm{C}\left(31^{\prime}\right)-\mathrm{C}\left(30^{\prime}\right)-\mathrm{H}(30 \mathrm{C})$ & 111.1 & $\mathrm{Co}(1)-\mathrm{C}(2)-\mathrm{H}(2 \mathrm{~B})$ & 109.5 \\
\hline $\mathrm{C}\left(31^{\prime}\right)-\mathrm{C}\left(30^{\prime}\right)-\mathrm{H}(30 \mathrm{D})$ & 111.1 & $\mathrm{Co}(1)-\mathrm{C}(2)-\mathrm{H}(2 \mathrm{C})$ & 109.5 \\
\hline $\mathrm{C}\left(30^{\prime}\right)-\mathrm{C}\left(31^{\prime}\right)-\mathrm{H}(31 \mathrm{C})$ & 111.9 & $\mathrm{H}(2 \mathrm{~A})-\mathrm{C}(2)-\mathrm{H}(2 \mathrm{~B})$ & 109.5 \\
\hline $\mathrm{C}\left(30^{\prime}\right)-\mathrm{C}\left(31^{\prime}\right)-\mathrm{H}(31 \mathrm{D})$ & 111.9 & $\mathrm{H}(2 \mathrm{~A})-\mathrm{C}(2)-\mathrm{H}(2 \mathrm{C})$ & 109.5 \\
\hline $\mathrm{H}(31 \mathrm{C})-\mathrm{C}\left(31^{\prime}\right)-\mathrm{H}(31 \mathrm{D})$ & 109.6 & $\mathrm{H}(2 \mathrm{~B})-\mathrm{C}(2)-\mathrm{H}(2 \mathrm{C})$ & 109.5 \\
\hline $\mathrm{N}\left(5^{\prime}\right)-\mathrm{C}\left(31^{\prime}\right)-\mathrm{C}\left(30^{\prime}\right)$ & $99.6(13)$ & $\mathrm{Co}(1)-\mathrm{C}\left(2^{\prime}\right)-\mathrm{H}\left(2^{\prime} \mathrm{A}\right)$ & 109.5 \\
\hline $\mathrm{N}\left(5^{\prime}\right)-\mathrm{C}\left(31^{\prime}\right)-\mathrm{H}(31 \mathrm{C})$ & 111.9 & $\mathrm{Co}(1)-\mathrm{C}\left(2^{\prime}\right)-\mathrm{H}\left(2^{\prime} \mathrm{B}\right)$ & 109.5 \\
\hline $\mathrm{N}\left(5^{\prime}\right)-\mathrm{C}\left(31^{\prime}\right)-\mathrm{H}(31 \mathrm{D})$ & 111.9 & $\mathrm{Co}(1)-\mathrm{C}\left(2^{\prime}\right)-\mathrm{H}\left(2^{\prime} \mathrm{C}\right)$ & 109.5 \\
\hline $\mathrm{C}\left(28^{\prime}\right)-\mathrm{N}\left(5^{\prime}\right)-\mathrm{C}\left(31^{\prime}\right)$ & $120.2(15)$ & $\mathrm{H}\left(2^{\prime} \mathrm{A}\right)-\mathrm{C}\left(2^{\prime}\right)-\mathrm{H}\left(2^{\prime} \mathrm{B}\right)$ & 109.5 \\
\hline $\mathrm{C}\left(28^{\prime}\right)-\mathrm{N}\left(5^{\prime}\right)-\mathrm{C}\left(32^{\prime}\right)$ & $116.4(17)$ & $\mathrm{H}\left(2^{\prime} \mathrm{A}\right)-\mathrm{C}\left(2^{\prime}\right)-\mathrm{H}\left(2^{\prime} \mathrm{C}\right)$ & 109.5 \\
\hline $\mathrm{C}\left(31^{\prime}\right)-\mathrm{N}\left(5^{\prime}\right)-\mathrm{C}\left(32^{\prime}\right)$ & $113.9(14)$ & $\mathrm{H}\left(2^{\prime} \mathrm{B}\right)-\mathrm{C}\left(2^{\prime}\right)-\mathrm{H}\left(2^{\prime} \mathrm{C}\right)$ & 109.5 \\
\hline $\mathrm{N}\left(5^{\prime}\right)-\mathrm{C}\left(32^{\prime}\right)-\mathrm{H}(32 \mathrm{D})$ & 109.5 & $\mathrm{H}(3 \mathrm{~A})-\mathrm{C}(3)-\mathrm{H}(3 \mathrm{~B})$ & 109.5 \\
\hline $\mathrm{N}\left(5^{\prime}\right)-\mathrm{C}\left(32^{\prime}\right)-\mathrm{H}(32 \mathrm{E})$ & 109.5 & $\mathrm{H}(3 \mathrm{~A})-\mathrm{C}(3)-\mathrm{H}(3 \mathrm{C})$ & 109.5 \\
\hline $\mathrm{N}\left(5^{\prime}\right)-\mathrm{C}\left(32^{\prime}\right)-\mathrm{H}(32 \mathrm{~F})$ & 109.5 & $\mathrm{H}(3 \mathrm{~B})-\mathrm{C}(3)-\mathrm{H}(3 \mathrm{C})$ & 109.5 \\
\hline $\mathrm{H}(32 \mathrm{D})-\mathrm{C}\left(32^{\prime}\right)-\mathrm{H}(32 \mathrm{E})$ & 109.5 & $\mathrm{C}(4)-\mathrm{C}(3)-\mathrm{H}(3 \mathrm{~A})$ & 109.5 \\
\hline $\mathrm{H}(32 \mathrm{D})-\mathrm{C}\left(32^{\prime}\right)-\mathrm{H}(32 \mathrm{~F})$ & 109.5 & $\mathrm{C}(4)-\mathrm{C}(3)-\mathrm{H}(3 \mathrm{~B})$ & 109.5 \\
\hline $\mathrm{H}(32 \mathrm{E})-\mathrm{C}\left(32^{\prime}\right)-\mathrm{H}(32 \mathrm{~F})$ & 109.5 & $\mathrm{C}(4)-\mathrm{C}(3)-\mathrm{H}(3 \mathrm{C})$ & 109.5 \\
\hline C(8)-N(1)-C(11) & $113.8(2)$ & $\mathrm{O}(1)-\mathrm{C}(4)-\mathrm{C}(3)$ & $117.2(2)$ \\
\hline $\mathrm{C}(8)-\mathrm{N}(1)-\mathrm{C}(12)$ & $124.6(2)$ & $\mathrm{O}(1)-\mathrm{C}(4)-\mathrm{C}(5)$ & $125.3(2)$ \\
\hline $\mathrm{C}(12)-\mathrm{N}(1)-\mathrm{C}(11)$ & $121.5(2)$ & $C(5)-C(4)-C(3)$ & $117.5(2)$ \\
\hline $\mathrm{C}(13)-\mathrm{N}(2)-\mathrm{C}(16)$ & $113.5(2)$ & $\mathrm{C}(4)-\mathrm{C}(5)-\mathrm{H}(5 \mathrm{~A})$ & 106.2 \\
\hline C(13)-N(2)-C(17) & $124.8(2)$ & $\mathrm{C}(4)-\mathrm{C}(5)-\mathrm{H}(5 \mathrm{~B})$ & 106.2 \\
\hline $\mathrm{C}(17)-\mathrm{N}(2)-\mathrm{C}(16)$ & $121.6(2)$ & $C(4)-C(5)-C(6)$ & $124.4(2)$ \\
\hline C(18)-N(3)-C(21) & $113.9(2)$ & $\mathrm{H}(5 \mathrm{~A})-\mathrm{C}(5)-\mathrm{H}(5 \mathrm{~B})$ & 106.4 \\
\hline $\mathrm{C}(18)-\mathrm{N}(3)-\mathrm{C}(22)$ & $124.2(2)$ & $\mathrm{C}(6)-\mathrm{C}(5)-\mathrm{H}(5 \mathrm{~A})$ & 106.2 \\
\hline $\mathrm{C}(22)-\mathrm{N}(3)-\mathrm{C}(21)$ & $121.8(2)$ & $\mathrm{C}(6)-\mathrm{C}(5)-\mathrm{H}(5 \mathrm{~B})$ & 106.2 \\
\hline $\mathrm{C}(23)-\mathrm{N}(4)-\mathrm{C}(26)$ & $113.9(2)$ & $\mathrm{O}(2)-\mathrm{C}(6)-\mathrm{C}(5)$ & $125.2(2)$ \\
\hline C(23)-N(4)-C(27) & $123.9(2)$ & $\mathrm{O}(2)-\mathrm{C}(6)-\mathrm{C}(7)$ & $116.9(2)$ \\
\hline C(27)-N(4)-C(26) & $121.6(2)$ & $C(5)-C(6)-C(7)$ & $117.9(2)$ \\
\hline $\mathrm{Co}(1)-\mathrm{C}(1)-\mathrm{H}(1 \mathrm{~A})$ & 109.5 & $\mathrm{C}(6)-\mathrm{C}(7)-\mathrm{H}(7 \mathrm{~A})$ & 109.5 \\
\hline $\mathrm{Co}(1)-\mathrm{C}(1)-\mathrm{H}(1 \mathrm{~B})$ & 109.5 & $\mathrm{C}(6)-\mathrm{C}(7)-\mathrm{H}(7 \mathrm{~B})$ & 109.5 \\
\hline $\mathrm{Co}(1)-\mathrm{C}(1)-\mathrm{H}(1 \mathrm{C})$ & 109.5 & $\mathrm{C}(6)-\mathrm{C}(7)-\mathrm{H}(7 \mathrm{C})$ & 109.5 \\
\hline $\mathrm{H}(1 \mathrm{~A})-\mathrm{C}(1)-\mathrm{H}(1 \mathrm{~B})$ & 109.5 & $\mathrm{H}(7 \mathrm{~A})-\mathrm{C}(7)-\mathrm{H}(7 \mathrm{~B})$ & 109.5 \\
\hline $\mathrm{H}(1 \mathrm{~A})-\mathrm{C}(1)-\mathrm{H}(1 \mathrm{C})$ & 109.5 & $\mathrm{H}(7 \mathrm{~A})-\mathrm{C}(7)-\mathrm{H}(7 \mathrm{C})$ & 109.5 \\
\hline $\mathrm{H}(1 \mathrm{~B})-\mathrm{C}(1)-\mathrm{H}(1 \mathrm{C})$ & 109.5 & $\mathrm{H}(7 \mathrm{~B})-\mathrm{C}(7)-\mathrm{H}(7 \mathrm{C})$ & 109.5 \\
\hline
\end{tabular}




\begin{tabular}{|c|c|c|c|}
\hline $\mathrm{O}(3)-\mathrm{C}(8)-\mathrm{N}(1)$ & $126.3(2)$ & $\mathrm{C}(14)-\mathrm{C}(15)-\mathrm{H}(15 \mathrm{~A})$ & 110.8 \\
\hline $\mathrm{O}(3)-\mathrm{C}(8)-\mathrm{C}(9)$ & $125.1(2)$ & $\mathrm{C}(14)-\mathrm{C}(15)-\mathrm{H}(15 \mathrm{~B})$ & 110.8 \\
\hline N(1)-C(8)-C(9) & $108.6(2)$ & $\mathrm{H}(15 \mathrm{~A})-\mathrm{C}(15)-\mathrm{H}(15 \mathrm{~B})$ & 108.9 \\
\hline $\mathrm{C}(8)-\mathrm{C}(9)-\mathrm{H}(9 \mathrm{~A})$ & 110.9 & $C(16)-C(15)-C(14)$ & $104.8(2)$ \\
\hline $\mathrm{C}(8)-\mathrm{C}(9)-\mathrm{H}(9 \mathrm{~B})$ & 110.9 & $\mathrm{C}(16)-\mathrm{C}(15)-\mathrm{H}(15 \mathrm{~A})$ & 110.8 \\
\hline$C(8)-C(9)-C(10)$ & $104.1(2)$ & $\mathrm{C}(16)-\mathrm{C}(15)-\mathrm{H}(15 \mathrm{~B})$ & 110.8 \\
\hline $\mathrm{H}(9 \mathrm{~A})-\mathrm{C}(9)-\mathrm{H}(9 \mathrm{~B})$ & 109.0 & $\mathrm{~N}(2)-\mathrm{C}(16)-\mathrm{C}(15)$ & $104.2(2)$ \\
\hline $\mathrm{C}(10)-\mathrm{C}(9)-\mathrm{H}(9 \mathrm{~A})$ & 110.9 & $\mathrm{~N}(2)-\mathrm{C}(16)-\mathrm{H}(16 \mathrm{~A})$ & 110.9 \\
\hline $\mathrm{C}(10)-\mathrm{C}(9)-\mathrm{H}(9 \mathrm{~B})$ & 110.9 & $\mathrm{~N}(2)-\mathrm{C}(16)-\mathrm{H}(16 \mathrm{~B})$ & 110.9 \\
\hline $\mathrm{C}(9)-\mathrm{C}(10)-\mathrm{H}(10 \mathrm{~A})$ & 110.9 & $\mathrm{C}(15)-\mathrm{C}(16)-\mathrm{H}(16 \mathrm{~A})$ & 110.9 \\
\hline $\mathrm{C}(9)-\mathrm{C}(10)-\mathrm{H}(10 \mathrm{~B})$ & 110.9 & $\mathrm{C}(15)-\mathrm{C}(16)-\mathrm{H}(16 \mathrm{~B})$ & 110.9 \\
\hline $\mathrm{H}(10 \mathrm{~A})-\mathrm{C}(10)-\mathrm{H}(10 \mathrm{~B})$ & 108.9 & $\mathrm{H}(16 \mathrm{~A})-\mathrm{C}(16)-\mathrm{H}(16 \mathrm{~B})$ & 108.9 \\
\hline $\mathrm{C}(11)-\mathrm{C}(10)-\mathrm{C}(9)$ & $104.2(2)$ & $\mathrm{N}(2)-\mathrm{C}(17)-\mathrm{H}(17 \mathrm{~A})$ & 109.5 \\
\hline $\mathrm{C}(11)-\mathrm{C}(10)-\mathrm{H}(10 \mathrm{~A})$ & 110.9 & $\mathrm{~N}(2)-\mathrm{C}(17)-\mathrm{H}(17 \mathrm{~B})$ & 109.5 \\
\hline $\mathrm{C}(11)-\mathrm{C}(10)-\mathrm{H}(10 \mathrm{~B})$ & 110.9 & $\mathrm{~N}(2)-\mathrm{C}(17)-\mathrm{H}(17 \mathrm{C})$ & 109.5 \\
\hline $\mathrm{N}(1)-\mathrm{C}(11)-\mathrm{C}(10)$ & $103.2(2)$ & $\mathrm{H}(17 \mathrm{~A})-\mathrm{C}(17)-\mathrm{H}(17 \mathrm{~B})$ & 109.5 \\
\hline $\mathrm{N}(1)-\mathrm{C}(11)-\mathrm{H}(11 \mathrm{~A})$ & 111.1 & $\mathrm{H}(17 \mathrm{~A})-\mathrm{C}(17)-\mathrm{H}(17 \mathrm{C})$ & 109.5 \\
\hline $\mathrm{N}(1)-\mathrm{C}(11)-\mathrm{H}(11 \mathrm{~B})$ & 111.1 & $\mathrm{H}(17 \mathrm{~B})-\mathrm{C}(17)-\mathrm{H}(17 \mathrm{C})$ & 109.5 \\
\hline $\mathrm{C}(10)-\mathrm{C}(11)-\mathrm{H}(11 \mathrm{~A})$ & 111.1 & $\mathrm{O}(5)-\mathrm{C}(18)-\mathrm{N}(3)$ & $123.6(2)$ \\
\hline $\mathrm{C}(10)-\mathrm{C}(11)-\mathrm{H}(11 \mathrm{~B})$ & 111.1 & $\mathrm{O}(5)-\mathrm{C}(18)-\mathrm{C}(19)$ & $127.7(2)$ \\
\hline $\mathrm{H}(11 \mathrm{~A})-\mathrm{C}(11)-\mathrm{H}(11 \mathrm{~B})$ & 109.1 & $\mathrm{~N}(3)-\mathrm{C}(18)-\mathrm{C}(19)$ & $108.7(2)$ \\
\hline $\mathrm{N}(1)-\mathrm{C}(12)-\mathrm{H}(12 \mathrm{~A})$ & 109.5 & $\mathrm{C}(18)-\mathrm{C}(19)-\mathrm{H}(19 \mathrm{~A})$ & 110.5 \\
\hline $\mathrm{N}(1)-\mathrm{C}(12)-\mathrm{H}(12 \mathrm{~B})$ & 109.5 & $\mathrm{C}(18)-\mathrm{C}(19)-\mathrm{H}(19 \mathrm{~B})$ & 110.5 \\
\hline $\mathrm{N}(1)-\mathrm{C}(12)-\mathrm{H}(12 \mathrm{C})$ & 109.5 & $\mathrm{C}(18)-\mathrm{C}(19)-\mathrm{H}(19 \mathrm{C})$ & 111.0 \\
\hline $\mathrm{H}(12 \mathrm{~A})-\mathrm{C}(12)-\mathrm{H}(12 \mathrm{~B})$ & 109.5 & $\mathrm{C}(18)-\mathrm{C}(19)-\mathrm{H}(19 \mathrm{D})$ & 111.0 \\
\hline $\mathrm{H}(12 \mathrm{~A})-\mathrm{C}(12)-\mathrm{H}(12 \mathrm{C})$ & 109.5 & $\mathrm{C}(18)-\mathrm{C}(19)-\mathrm{C}\left(20^{\prime}\right)$ & $103.6(3)$ \\
\hline $\mathrm{H}(12 \mathrm{~B})-\mathrm{C}(12)-\mathrm{H}(12 \mathrm{C})$ & 109.5 & $\mathrm{H}(19 \mathrm{~A})-\mathrm{C}(19)-\mathrm{H}(19 \mathrm{~B})$ & 108.7 \\
\hline $\mathrm{O}(4)-\mathrm{C}(13)-\mathrm{N}(2)$ & $123.4(2)$ & $\mathrm{H}(19 \mathrm{C})-\mathrm{C}(19)-\mathrm{H}(19 \mathrm{D})$ & 109.0 \\
\hline $\mathrm{O}(4)-\mathrm{C}(13)-\mathrm{C}(14)$ & $127.3(2)$ & $\mathrm{C}(20)-\mathrm{C}(19)-\mathrm{C}(18)$ & $106.2(2)$ \\
\hline N(2)-C(13)-C(14) & $109.3(2)$ & $\mathrm{C}(20)-\mathrm{C}(19)-\mathrm{H}(19 \mathrm{~A})$ & 110.5 \\
\hline $\mathrm{C}(13)-\mathrm{C}(14)-\mathrm{H}(14 \mathrm{~A})$ & 110.8 & $\mathrm{C}(20)-\mathrm{C}(19)-\mathrm{H}(19 \mathrm{~B})$ & 110.5 \\
\hline $\mathrm{C}(13)-\mathrm{C}(14)-\mathrm{H}(14 \mathrm{~B})$ & 110.8 & $\mathrm{C}\left(20^{\prime}\right)-\mathrm{C}(19)-\mathrm{H}(19 \mathrm{C})$ & 111.0 \\
\hline$C(13)-C(14)-C(15)$ & $104.7(2)$ & $\mathrm{C}\left(20^{\prime}\right)-\mathrm{C}(19)-\mathrm{H}(19 \mathrm{D})$ & 111.0 \\
\hline $\mathrm{H}(14 \mathrm{~A})-\mathrm{C}(14)-\mathrm{H}(14 \mathrm{~B})$ & 108.9 & $\mathrm{C}(19)-\mathrm{C}(20)-\mathrm{H}(20 \mathrm{~A})$ & 111.1 \\
\hline $\mathrm{C}(15)-\mathrm{C}(14)-\mathrm{H}(14 \mathrm{~A})$ & 110.8 & $\mathrm{C}(19)-\mathrm{C}(20)-\mathrm{H}(20 \mathrm{~B})$ & 111.1 \\
\hline $\mathrm{C}(15)-\mathrm{C}(14)-\mathrm{H}(14 \mathrm{~B})$ & 110.8 & $C(19)-C(20)-C(21)$ & $103.3(3)$ \\
\hline
\end{tabular}




\begin{tabular}{|c|c|c|c|}
\hline $\mathrm{H}(20 \mathrm{~A})-\mathrm{C}(20)-\mathrm{H}(20 \mathrm{~B})$ & 109.1 & $\mathrm{C}(24)-\mathrm{C}(25)-\mathrm{H}(25 \mathrm{~A})$ & 110.8 \\
\hline $\mathrm{C}(21)-\mathrm{C}(20)-\mathrm{H}(20 \mathrm{~A})$ & 111.1 & $\mathrm{C}(24)-\mathrm{C}(25)-\mathrm{H}(25 \mathrm{~B})$ & 110.8 \\
\hline $\mathrm{C}(21)-\mathrm{C}(20)-\mathrm{H}(20 \mathrm{~B})$ & 111.1 & $\mathrm{H}(25 \mathrm{~A})-\mathrm{C}(25)-\mathrm{H}(25 \mathrm{~B})$ & 108.9 \\
\hline $\mathrm{C}(19)-\mathrm{C}\left(20^{\prime}\right)-\mathrm{H}(20 \mathrm{C})$ & 110.9 & $\mathrm{C}(26)-\mathrm{C}(25)-\mathrm{C}(24)$ & $104.6(2)$ \\
\hline $\mathrm{C}(19)-\mathrm{C}\left(20^{\prime}\right)-\mathrm{H}(20 \mathrm{D})$ & 110.9 & $\mathrm{C}(26)-\mathrm{C}(25)-\mathrm{H}(25 \mathrm{~A})$ & 110.8 \\
\hline $\mathrm{H}(20 \mathrm{C})-\mathrm{C}\left(20^{\prime}\right)-\mathrm{H}(20 \mathrm{D})$ & 108.9 & $\mathrm{C}(26)-\mathrm{C}(25)-\mathrm{H}(25 \mathrm{~B})$ & 110.8 \\
\hline $\mathrm{C}(21)-\mathrm{C}\left(20^{\prime}\right)-\mathrm{C}(19)$ & $104.2(4)$ & $\mathrm{N}(4)-\mathrm{C}(26)-\mathrm{C}(25)$ & $103.1(2)$ \\
\hline $\mathrm{C}(21)-\mathrm{C}\left(20^{\prime}\right)-\mathrm{H}(20 \mathrm{C})$ & 110.9 & $\mathrm{~N}(4)-\mathrm{C}(26)-\mathrm{H}(26 \mathrm{~A})$ & 111.1 \\
\hline $\mathrm{C}(21)-\mathrm{C}\left(20^{\prime}\right)-\mathrm{H}(20 \mathrm{D})$ & 110.9 & $\mathrm{~N}(4)-\mathrm{C}(26)-\mathrm{H}(26 \mathrm{~B})$ & 111.1 \\
\hline $\mathrm{N}(3)-\mathrm{C}(21)-\mathrm{C}(20)$ & $103.4(2)$ & $\mathrm{C}(25)-\mathrm{C}(26)-\mathrm{H}(26 \mathrm{~A})$ & 111.1 \\
\hline $\mathrm{N}(3)-\mathrm{C}(21)-\mathrm{C}\left(20^{\prime}\right)$ & $103.3(3)$ & $\mathrm{C}(25)-\mathrm{C}(26)-\mathrm{H}(26 \mathrm{~B})$ & 111.1 \\
\hline $\mathrm{N}(3)-\mathrm{C}(21)-\mathrm{H}(21 \mathrm{~A})$ & 111.1 & $\mathrm{H}(26 \mathrm{~A})-\mathrm{C}(26)-\mathrm{H}(26 \mathrm{~B})$ & 109.1 \\
\hline $\mathrm{N}(3)-\mathrm{C}(21)-\mathrm{H}(21 \mathrm{~B})$ & 111.1 & $\mathrm{~N}(4)-\mathrm{C}(27)-\mathrm{H}(27 \mathrm{~A})$ & 109.5 \\
\hline $\mathrm{N}(3)-\mathrm{C}(21)-\mathrm{H}(21 \mathrm{C})$ & 111.1 & $\mathrm{~N}(4)-\mathrm{C}(27)-\mathrm{H}(27 \mathrm{~B})$ & 109.5 \\
\hline $\mathrm{N}(3)-\mathrm{C}(21)-\mathrm{H}(21 \mathrm{D})$ & 111.1 & $\mathrm{~N}(4)-\mathrm{C}(27)-\mathrm{H}(27 \mathrm{C})$ & 109.5 \\
\hline $\mathrm{C}(20)-\mathrm{C}(21)-\mathrm{H}(21 \mathrm{~A})$ & 111.1 & $\mathrm{H}(27 \mathrm{~A})-\mathrm{C}(27)-\mathrm{H}(27 \mathrm{~B})$ & 109.5 \\
\hline $\mathrm{C}(20)-\mathrm{C}(21)-\mathrm{H}(21 \mathrm{~B})$ & 111.1 & $\mathrm{H}(27 \mathrm{~A})-\mathrm{C}(27)-\mathrm{H}(27 \mathrm{C})$ & 109.5 \\
\hline $\mathrm{C}\left(20^{\prime}\right)-\mathrm{C}(21)-\mathrm{H}(21 \mathrm{C})$ & 111.1 & $\mathrm{H}(27 \mathrm{~B})-\mathrm{C}(27)-\mathrm{H}(27 \mathrm{C})$ & 109.5 \\
\hline $\mathrm{C}\left(20^{\prime}\right)-\mathrm{C}(21)-\mathrm{H}(21 \mathrm{D})$ & 111.1 & & \\
\hline $\mathrm{H}(21 \mathrm{~A})-\mathrm{C}(21)-\mathrm{H}(21 \mathrm{~B})$ & 109.1 & & \\
\hline $\mathrm{H}(21 \mathrm{C})-\mathrm{C}(21)-\mathrm{H}(21 \mathrm{D})$ & 109.1 & & \\
\hline $\mathrm{N}(3)-\mathrm{C}(22)-\mathrm{H}(22 \mathrm{~A})$ & 109.5 & & \\
\hline $\mathrm{N}(3)-\mathrm{C}(22)-\mathrm{H}(22 \mathrm{~B})$ & 109.5 & & \\
\hline $\mathrm{N}(3)-\mathrm{C}(22)-\mathrm{H}(22 \mathrm{C})$ & 109.5 & & \\
\hline $\mathrm{H}(22 \mathrm{~A})-\mathrm{C}(22)-\mathrm{H}(22 \mathrm{~B})$ & 109.5 & & \\
\hline $\mathrm{H}(22 \mathrm{~A})-\mathrm{C}(22)-\mathrm{H}(22 \mathrm{C})$ & 109.5 & & \\
\hline $\mathrm{H}(22 \mathrm{~B})-\mathrm{C}(22)-\mathrm{H}(22 \mathrm{C})$ & 109.5 & & \\
\hline $\mathrm{O}(6)-\mathrm{C}(23)-\mathrm{N}(4)$ & $124.3(2)$ & & \\
\hline $\mathrm{O}(6)-\mathrm{C}(23)-\mathrm{C}(24)$ & $126.7(2)$ & & \\
\hline $\mathrm{N}(4)-\mathrm{C}(23)-\mathrm{C}(24)$ & $109.0(2)$ & & \\
\hline $\mathrm{C}(23)-\mathrm{C}(24)-\mathrm{H}(24 \mathrm{~A})$ & 111.0 & & \\
\hline $\mathrm{C}(23)-\mathrm{C}(24)-\mathrm{H}(24 \mathrm{~B})$ & 111.0 & & \\
\hline$C(23)-C(24)-C(25)$ & $103.7(2)$ & & \\
\hline $\mathrm{H}(24 \mathrm{~A})-\mathrm{C}(24)-\mathrm{H}(24 \mathrm{~B})$ & 109.0 & & \\
\hline $\mathrm{C}(25)-\mathrm{C}(24)-\mathrm{H}(24 \mathrm{~A})$ & 111.0 & & \\
\hline $\mathrm{C}(25)-\mathrm{C}(24)-\mathrm{H}(24 \mathrm{~B})$ & 111.0 & & \\
\hline
\end{tabular}


Symmetry transformations used to generate equivalent atoms:

\#1 -x,-y+1,-z+1 